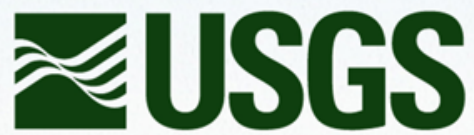

science for a changing world

Prepared in cooperation with the city of Rittman and the Muskingum Watershed Conservancy District

\title{
Hydrologic and Hydraulic Analyses of Selected Streams near the City of Rittman in Wayne and Medina Counties, Ohio
}

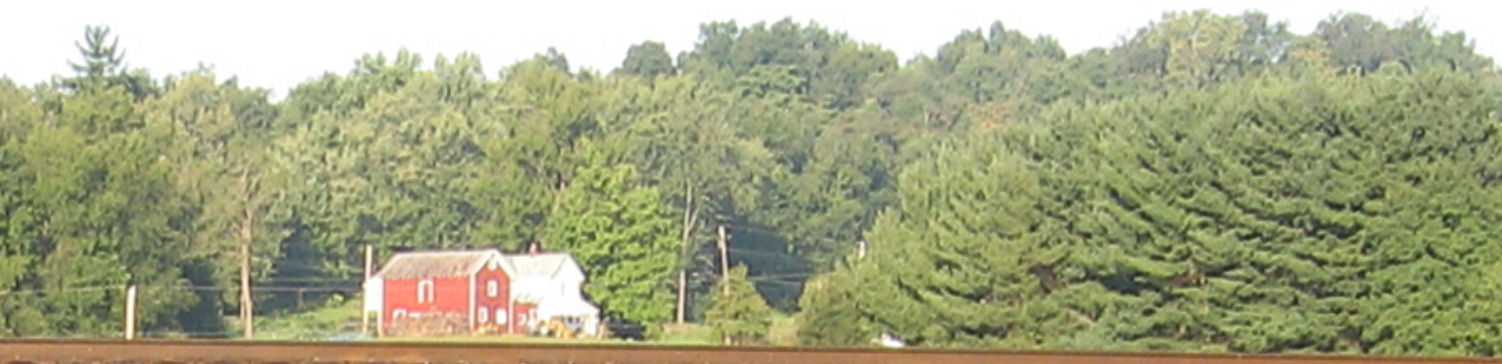

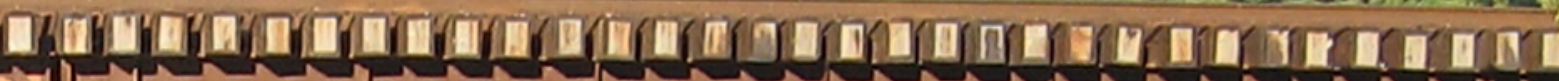
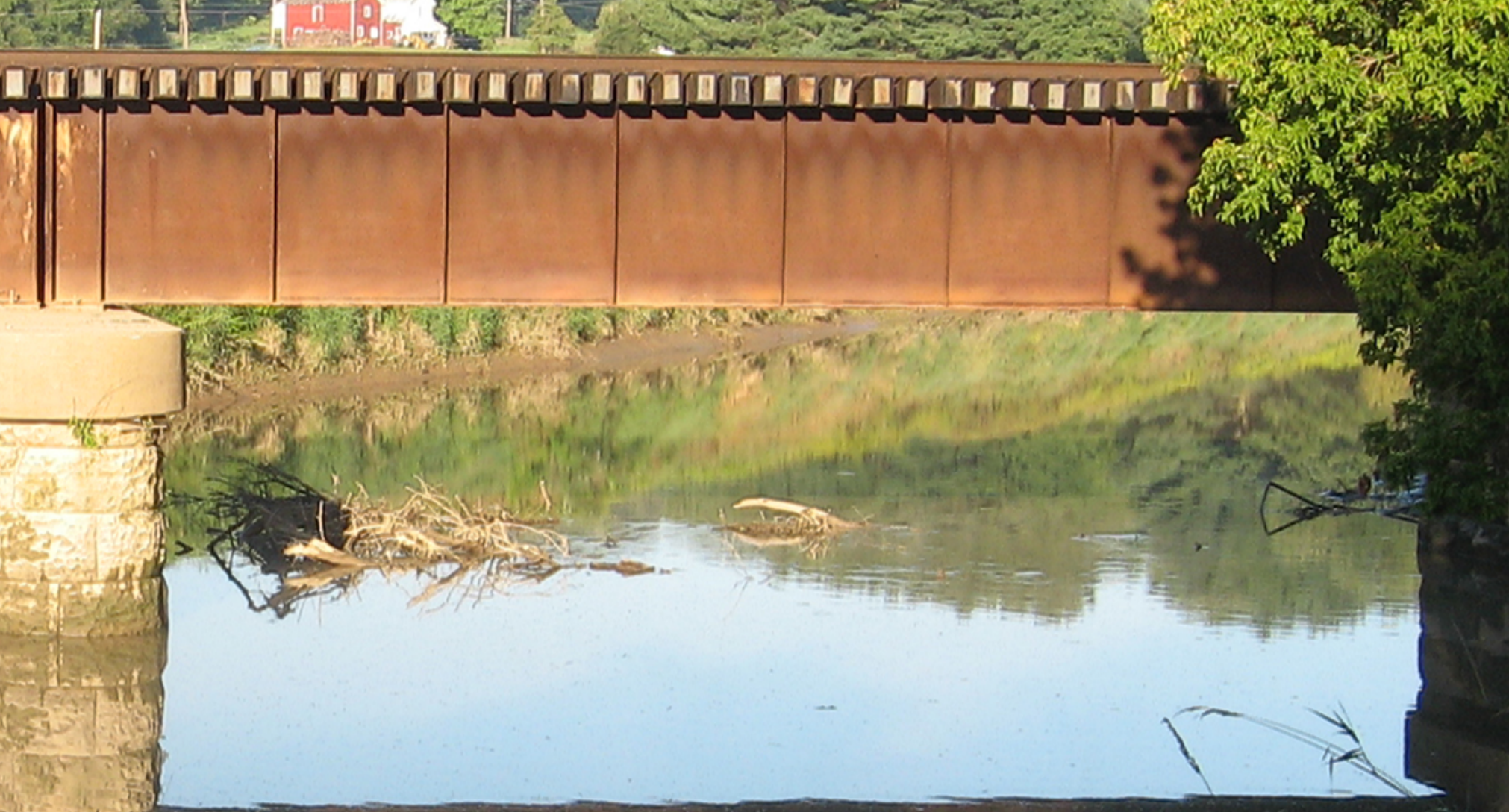

Scientific Investigations Report 2021-5040

A ${ }^{\prime}$ U.S. Department of the Interior U.S. Geological Survey 
Front, back covers. View looking upstream at the CSX transportation railroad bridge over Chippewa Creek just upstream from the confluence with the Tuscarawas River near Clinton, Ohio (Photograph by U.S. Geological Survey, September 2009). 


\section{Hydrologic and Hydraulic Analyses of Selected Streams near the City of Rittman in Wayne and Medina Counties, Ohio}

By Chad J. Ostheimer

Prepared in cooperation with the city of Rittman and the Muskingum Watershed Conservancy District

Scientific Investigations Report 2021-5040 


\section{U.S. Geological Survey, Reston, Virginia: 2021}

For more information on the USGS - the Federal source for science about the Earth, its natural and living resources, natural hazards, and the environment—visit https://www.usgs.gov or call 1-888-ASK-USGS.

For an overview of USGS information products, including maps, imagery, and publications, visit https://store.usgs.gov/.

Any use of trade, firm, or product names is for descriptive purposes only and does not imply endorsement by the U.S. Government.

Although this information product, for the most part, is in the public domain, it also may contain copyrighted materials as noted in the text. Permission to reproduce copyrighted items must be secured from the copyright owner.

Suggested citation:

Ostheimer, C.J., 2021, Hydrologic and hydraulic analyses of selected streams near the city of Rittman in Wayne and Medina Counties, Ohio: U.S. Geological Survey Scientific Investigations Report 2021-5040, 30 p.,

https://doi.org/10.3133/sir20215040.

Associated data for this publication:

Ostheimer, C.J., 2021, Geospatial datasets and hydraulic models for selected streams near Rittman in Wayne and Medina Counties, Ohio: U.S. Geological Survey data release, https://doi.org/10.5066/P9W6ROMC.

ISSN 2328-0328 (online) 


\section{Contents}

Abstract

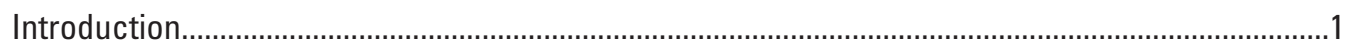

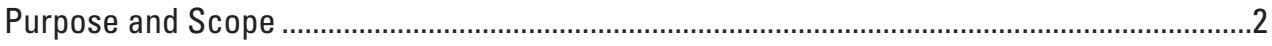

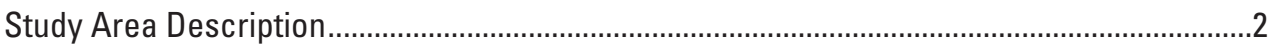

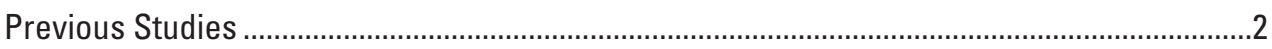

Study Approach

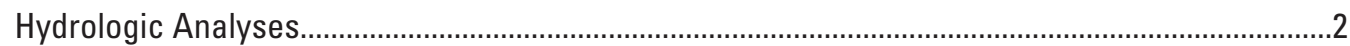

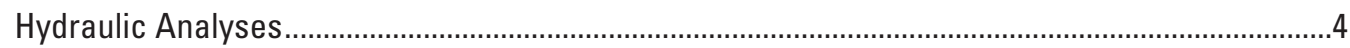

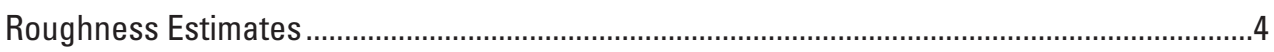

Field Surveys

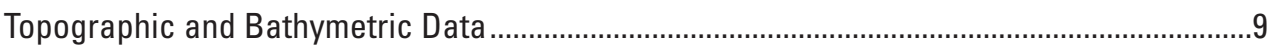

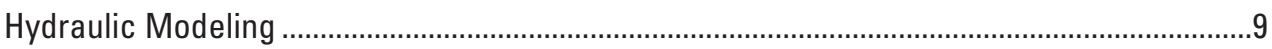

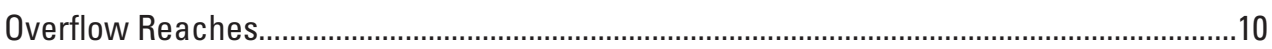

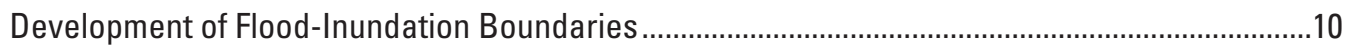

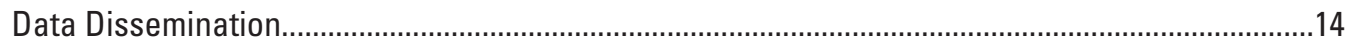

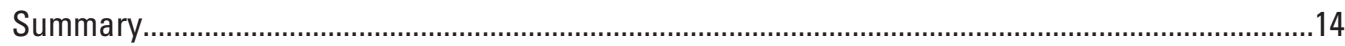

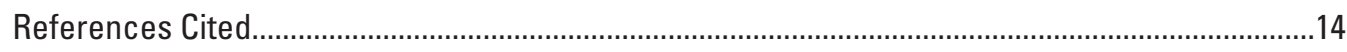

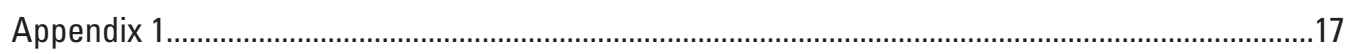

\section{Figures}

1. Map showing locations of study reaches and U.S. Geological Survey streamgages in Wayne and Medina Counties, Ohio ......

2. Map showing locations of flow path and junctions at which flow leaves and returns to the main channel of Chippewa Creek

3. Map showing locations of flow paths and points at which flow leaves and returns to the main channel of the unnamed tributary to Styx River

\section{Tables}

1. Summary of the explanatory variable values used in the regression equations, the initial 10-, 4-, 2-, 1-, and 0.2-percent and 1-percent plus annual exceedance probability flood-peak streamflow estimates, and locations for the selected streams in Wayne and Medina Counties, Ohio

2. Selected characteristics of U.S. Geological Survey gages within the Chippewa Creek drainage area in Wayne and Medina Counties, Ohio

3. Peak-flood streamflow estimates for the 10-, 4-, 2-, 1-, and 0.2-percent annual exceedance probability floods as reported in Koltun, where $R$ is equal to the weighted estimate divided by the regression estimate

4. Summary of the final 10-, 4-, 2-, 1-, and 0.2-percent and 1-percent plus annual exceedance probability flood-peak streamflow estimates and locations for the selected streams in Wayne and Medina Counties, Ohio 
5. Survey characteristics and hydraulic parameters used in the hydraulic models............9

6. Streamflows resulting in balanced water-surface elevations between the main stem of Chippewa Creek and the overflow reach or Westfield Ditch

7. Streamflows resulting in balanced water-surface elevations between the main stem of the unnamed tributary to Styx River and the overflow reaches.

\section{Conversion Factors}

U.S. customary units to International System of Units

\begin{tabular}{lcl}
\hline \multicolumn{1}{c}{ Multiply } & By & \multicolumn{1}{c}{ To obtain } \\
\hline & Length & \\
\hline foot (ft) & 0.3048 & meter $(\mathrm{m})$ \\
inch (in.) & 25.4 & millimeter $(\mathrm{mm})$ \\
mile (mi) & 1.609 & kilometer $(\mathrm{km})$ \\
\hline & Area & \\
\hline square mile $\left(\mathrm{mi}^{2}\right)$ & 2.590 & square kilometer $\left(\mathrm{km}^{2}\right)$ \\
\hline & Flow rate & \\
\hline cubic foot per second $\left(\mathrm{ft}^{3} / \mathrm{s}\right)$ & 0.02832 & cubic meter per second $\left(\mathrm{m}^{3} / \mathrm{s}\right)$ \\
\hline
\end{tabular}

\section{Datum}

Vertical coordinate information for stage is referenced to the height above an arbitrary datum established at a streamgage, and vertical coordinate information for elevation is referenced to the height above the North American Vertical Datum of 1988 (NAVD 88) and [or] the National Geodetic Vertical Datum of 1929 (NGVD 29).

Horizontal coordinate information is referenced to the North American Datum of 1983 (NAD 83).

\section{Abbreviations}

$\begin{array}{ll}\text { AEP } & \text { annual exceedance probability } \\ \text { DEM } & \text { digital elevation model } \\ \text { DGPS } & \text { differential global positioning system } \\ \text { FEMA } & \text { Federal Emergency Management Agency } \\ \text { FIS } & \text { Flood Insurance Study } \\ \text { HEC-GeoRAS } & \text { Hydrologic Engineering Center Geospatial River Analysis System } \\ \text { HEC-RAS } & \text { Hydrologic Engineering Center River Analysis System } \\ \text { lidar } & \text { light detection and ranging } \\ \text { USGS } & \text { U.S. Geological Survey }\end{array}$




\title{
Hydrologic and Hydraulic Analyses of Selected Streams near the City of Rittman in Wayne and Medina Counties, Ohio
}

\author{
By Chad J. Ostheimer
}

\section{Abstract}

The U.S. Geological Survey, in cooperation with the Muskingum Watershed Conservancy District and the city of Rittman, Ohio, did a study to provide data to update and expand parts of two Federal Emergency Management Agency Flood Insurance Studies. The study consisted of hydrologic and hydraulic analyses for selected reaches of four streams (Chippewa Creek, Little Chippewa Creek, Styx River, and the unnamed tributary to Styx River) near the city of Rittman in Wayne and Medina Counties, Ohio. The study covered 36.2 miles of stream reaches.

Instantaneous peak streamflows for floods with 10-, 4-, 2-, 1-, and 0.2-percent and 1-percent plus annual exceedance probabilities were estimated using historical streamflow data from three U.S. Geological Survey streamgages and regional flood-frequency regression equations. The flood-frequency estimates were then used in a Hydrologic Engineering Center River Analysis System step-backwater model to determine water-surface profiles; flood-inundation boundaries for the 10-, 4-, 2-, 1-, and 0.2-percent and 1-percent plus annual exceedance probabilities; and a regulatory floodway for the study reaches. Model inputs included cross sections derived from a digital elevation model supplemented with field surveys of open-channel cross sections and hydraulic structures, field estimates of Manning's roughness values, and flood estimates determined from regional regression equations and historical streamflow data. Flood-inundation boundaries were mapped for each stream reach for the 1- and 0.2-percent annual exceedance probability floods and a regulatory floodway. All data used in the creation of the flood-inundation boundaries are available through a U.S. Geological Survey data release (Ostheimer, 2021) and will be submitted to the Federal Emergency Management Agency for inclusion in updated Flood Insurance Studies for Wayne and Medina Counties.

\section{Introduction}

Several streams within the Chippewa Creek Basin in Wayne and Medina Counties, Ohio, were damaged by flooding in 1913, 1935, 1950, 1959, 1969, 2003, and 2019. The July 1969 flood on Chippewa Creek in Rittman, Ohio, had an estimated annual exceedance probability (AEP) of 1-percent (a recurrence interval of 100 years) (Federal Emergency Management Agency, 2009).

Before this study, officials and emergency responders relied on several information sources to make decisions on how to best alert the public to potential flood hazards and mitigate flood damages. These sources include:

1. the Federal Emergency Management Agency (FEMA) Flood Insurance Study (FIS) for Wayne County, Ohio, dated August 18, 2009 (Federal Emergency Management Agency, 2009;

2. the FEMA FIS for Medina County, Ohio, dated August 19, 2013 (Federal Emergency Management Agency, 2013); and

3. the U.S. Geological Survey (USGS) National Water Information System database from which current (U.S. Geological Survey, 2020a, b, c) and historical (U.S. Geological Survey, 2020d) stages and streamflows, including annual peak streamflows, can be obtained for streams in the study area.

After the 2019 flooding in Rittman, Ohio, the USGS began a study in cooperation with the city of Rittman, Ohio, and the Muskingum Watershed Conservancy District to update and expand the FEMA FIS for Wayne and Medina Counties, Ohio. 


\section{Purpose and Scope}

The purpose of this report is to describe the methods used to carry out hydrologic and hydraulic analyses for four stream reaches in Wayne and Medina Counties, Ohio, totaling 36.2 miles in length (fig. 1) and the results of those analyses. The analyses include (1) estimates of flood-peak streamflows corresponding to floods with AEPs of 10-, 4-, 2-, 1-, and 0.2 -percent and 1-percent plus and (2) determination of watersurface profiles and flood-plain boundaries associated with the AEPs and a regulatory floodway. Streamflows with AEPs of $10,4,2,1$, and 0.2 percent correspond to recurrence intervals of $10,25,50,100$, and 500 years, respectively.

\section{Study Area Description}

The northeast Ohio counties of Wayne and Medina had 2019 estimated populations of approximately 115,700 (U.S. Census Bureau, 2010a) and 179,700 (U.S. Census Bureau, 2010b), respectively. This study's focus is near the city of Rittman, which is a small town of approximately 6,500 residents (U.S. Census Bureau, 2010c) in northeast Wayne County and encompasses the confluences of the unnamed tributary to Styx River with the Styx River, Styx River with Chippewa Creek, and Little Chippewa Creek with Chippewa Creek. The streams evaluated in this study lie within the Chippewa Creek Basin and (except for Little Chippewa Creek which drains northeast) drain mainly to the south and east. The 1981 to 2010 mean annual precipitation in the Chippewa Creek Basin was 38.3 inches as determined from Parameter-Elevation Regressions on Independent Slopes Model datasets (PRISM Climate Group, 2012). Land cover in the Chippewa Creek Basin is predominately planted or cultivated land (61 percent), with the rest of the basin having mostly developed or forested land covers (19 and 17 percent, respectively) (U.S. Geological Survey, 2014). Development within the Chippewa Creek Basin is concentrated in several pockets that are surrounded by large rural expanses.

\section{Previous Studies}

The effective FEMA FIS for Wayne County (Federal Emergency Management Agency, 2009) was published on August 18, 2009, while the effective FEMA FIS for Medina County (Federal Emergency Management Agency, 2013) was published on August 19, 2013. The detailed hydrologic and hydraulic analyses in the 2009 FIS and 2013 FIS (for all four stream reaches) were completed in the 1980s. Little Chippewa Creek has not been studied using detailed hydraulic methods.

\section{Study Approach}

The study included the following elements:

1. collection of topographic and bathymetric data for selected cross sections and geometric data for structures and bridges along the study reaches;

2. estimation of 10-, 4-, 2-, 1-, and 0.2-percent AEP peak streamflows using regional regression equations and streamgage data (where available);

3. estimation of energy-loss factors (Manning's roughness coefficients) for the stream channel and flood plain;

4. computation of flood profiles (including a regulatory floodway) using the U.S. Army Corps of Engineers Hydrologic Engineering Center's (HEC) River Analysis System (HEC-RAS) computer program (U.S. Army Corps of Engineers, 2016);

5. production of flood-inundation maps, initially using the HEC Geospatial River Analysis System (HEC-GeoRAS computer program (U.S. Army Corps of Engineers, 2009) and then refined with a geographic information system; and

6. submission of flood profile and regulatory floodway data to FEMA for inclusion in revised floodinsurance studies.

\section{Hydrologic Analyses}

Regional regression equations were used to estimate the 10-, 4-, 2-, 1-, and 0.2-percent and 1-percent plus AEP flood-peak streamflows for selected locations (generally at stream confluences or at easily identifiable points [such as a road crossing] along stream reaches with gradually increasing drainage area) on all stream reaches. The 1-percent plus flood elevation is defined as a flood elevation derived using streamflows that are at the upper 84-percent confidence limit as calculated in the streamgage analysis for the 1-percent AEP flood (Federal Emergency Management Agency, 2019). 


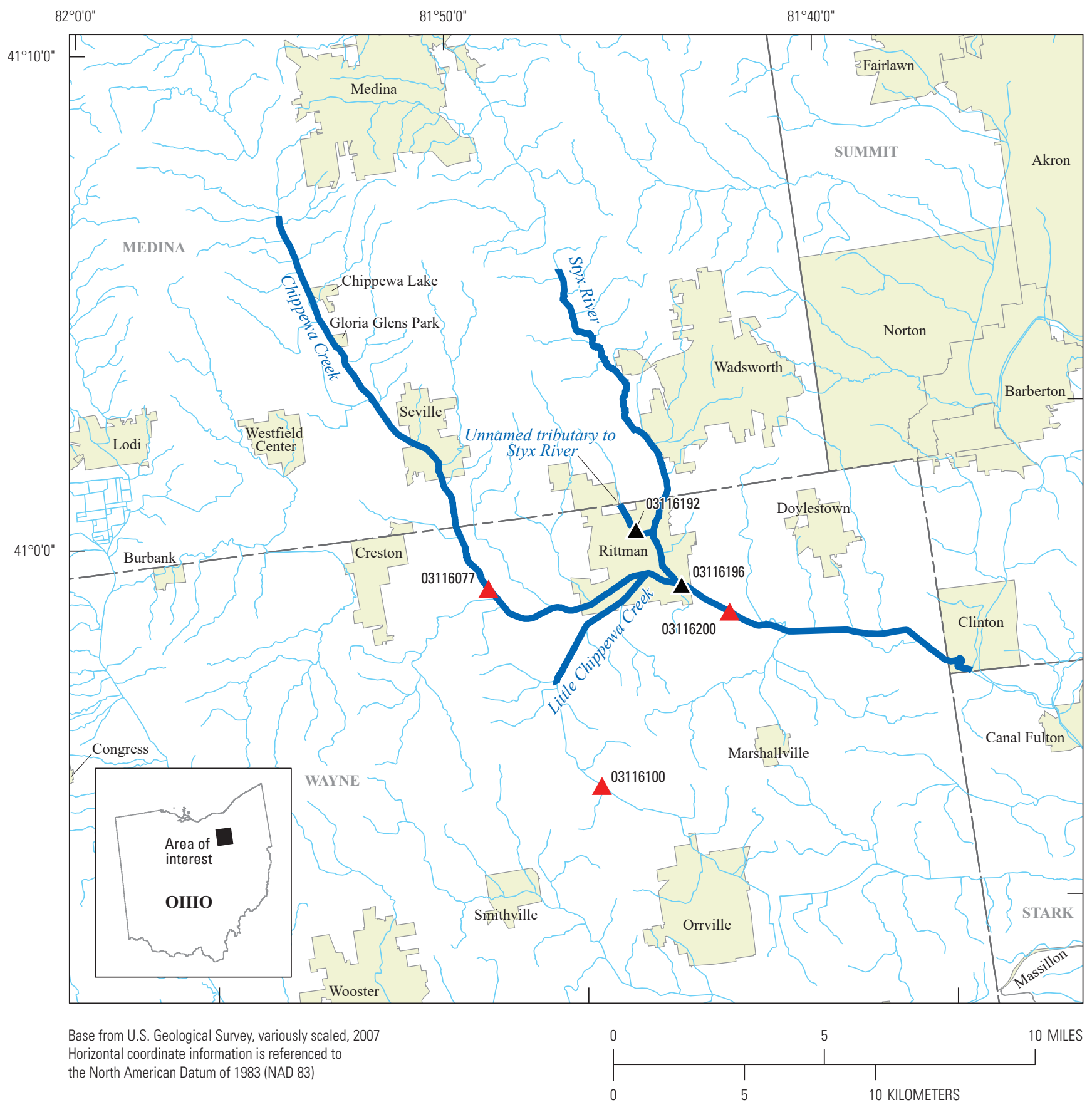

EXPLANATION

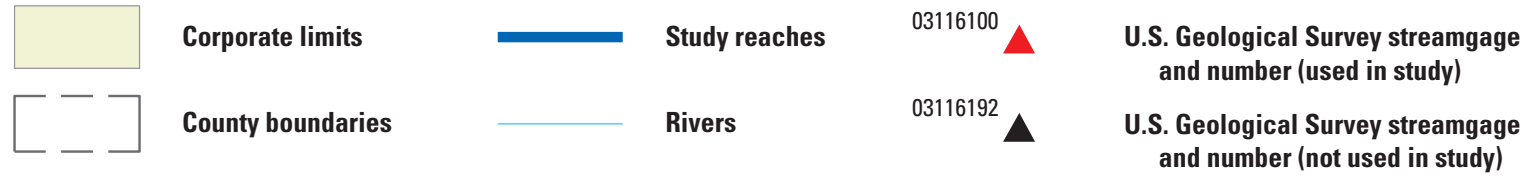

Figure 1. Locations of study reaches and U.S. Geological Survey streamgages in Wayne and Medina Counties, Ohio. 
The Ohio StreamStats application (Koltun, 2019) was used to solve regional regression equations that use (1) drainage area, (2) main channel slope ([SL10-85] determined by the 10-85 method), and (3) storage (percentage of drainage classified as water and wetlands area) as explanatory variables. The explanatory variables are computed within StreamStats for user-defined locations from geospatial datasets. StreamStats estimates assume that the basin is not appreciably regulated and is without substantial urbanization (Koltun, 2019). The resulting initial flood-peak estimates are listed in table 1.

Of the five USGS streamgages in the study area (table 2), three streamgages (Chippewa Creek at Miller Road at Sterling, Ohio [U.S. Geological Survey station number 03116077], Little Chippewa Creek near Smithville, Ohio [U.S. Geological Survey station number 03116100], and Chippewa Creek at Easton, Ohio [U.S. Geological Survey station number 03116200]), have sufficient peak-flow record (greater than or equal to 10 years) to estimate flood-frequency statistics (Interagency Advisory Committee on Water Data, 1982). Flood-frequency statistics were not computed for the unnamed tributary to Styx River at Rittman, Ohio (03116192), or Chippewa Creek above Easton, Ohio (03116196), due to lack of streamflow data and insufficient streamflow record, respectively. Hereafter, the USGS streamgages Chippewa Creek at Miller Road at Sterling, Ohio (03116077), and Chippewa Creek at Easton, Ohio (03116200), will be referred to as the Sterling and Easton streamgages, respectively.

Flood-frequency estimates determined with StreamStats for ungaged locations on Chippewa Creek were subsequently adjusted using the gage-weighting technique described by Koltun (2019). For the 1-percent plus flood, the $R$ factor from equation 15 in Koltun (2019) was assumed to be the same as for the 1-percent AEP flood. The data used to determine the $R$ factors are shown in table 3 .

For Chippewa Creek, the gage-weighting process resulted in logically increasing flood-peak streamflows with increasing drainage area for locations between the Sterling and Easton streamgages. However, for locations upstream from the Sterling streamgage, the gage-weighting process resulted in flood-peak streamflows increasing with decreasing drainage area. Likewise, downstream from the Easton streamgage, the gage-weighting process resulted in flood-peak streamflows decreasing with increasing drainage area. Consequently, a different method was used to estimate flood-frequency statistics at ungaged locations in those reaches. Koltun (2019) provides equations for estimating flood-frequency statistics, using drainage area as the only explanatory variable. These are referred to as "drainage-area only" equations. To produce a logical progression of flood-frequency estimates for locations upstream from the Sterling streamgage and downstream from the Easton streamgage, the gage-weighted estimate at the nearest streamgage was multiplied by the ratio of the drainage-area only estimate at the ungaged locations to the drainage-area only estimate at the streamgage location. This ratio adjustment technique resulted in flood-frequency estimates that monotonically decreased upstream from the Sterling streamgage and increased downstream from the Easton streamgage at rates approximately equal to those indicated by the drainage-area only equations. The final adjusted flood-frequency estimates are given in table 4 .

\section{Hydraulic Analyses}

The water-surface profiles used to develop the floodinundation maps for this study were computed using HEC-RAS, version 5.0.3 (U.S. Army Corps of Engineers, 2016). HEC-RAS can perform one- and two-dimensional hydraulic calculations for a network of channels under steady- or unsteady-state streamflow conditions. All profiles in this study were computed using one-dimensional steady-state streamflow calculations. Inputs for steady-state streamflow calculations include streamflow regime, boundary conditions, selected hydraulic characteristics, and streamflow estimates. A subcritical streamflow regime was indicated and used for all calculations.

\section{Roughness Estimates}

Hydraulic analyses require the estimation of energy losses exerted by a channel on flow. These energy losses are quantified by Manning's roughness coefficient (" $n$ " value). The $n$ values were selected on the basis of field observations and high-resolution aerial photographs. For the study reaches, the $n$ values ranged from 0.010 to 0.049 (open lake to riprap/debris piles) for the main channel and from 0.028 to 0.090 (short grass to heavily wooded) for the overbank areas (table 5).

\section{Field Surveys}

The USGS used a differential global positioning system (DGPS) and differential-leveling surveys (hereafter referred to as conventional surveys) for this study. The DGPS surveys were used to establish an elevation control network at pertinent locations along each of the streams studied. Conventional surveys were done to obtain stream and hydraulic-structure geometry. All conventional survey data met third-order accuracy (horizontal and vertical) criteria (Federal Geodetic Control Committee, 1984). DGPS surveys were completed using real-time network surveying techniques (Rydlund and Densmore, 2012). Elevations determined using the DGPS 
Table 1. Summary of the explanatory variable values used in the regression equations, the initial 10-, 4-, 2-, 1-, and 0.2-percent and 1-percent plus ${ }^{1}$ annual exceedance probability flood-peak streamflow estimates, and locations for the selected streams in Wayne and Medina Counties, Ohio.

$\left[\mathrm{mi}^{2}\right.$, square mile; ft/mi, foot per mile; $\mathrm{ft}^{3} / \mathrm{s}$, cubic foot per second; \%, percent; US, upstream; ${ }^{\circ}$, degree; ', minute; ", second; approx, approximately; ft, foot; Rd, Road; USGS, U.S. Geological Survey; DS, downstream; S, South; St, Street]

\begin{tabular}{|c|c|c|c|c|c|c|c|c|c|c|c|}
\hline \multirow[b]{2}{*}{ Location description } & \multirow[b]{2}{*}{ Latitude } & \multirow[b]{2}{*}{ Longitude } & \multirow{2}{*}{$\begin{array}{l}\text { Drainage } \\
\text { area }\left(\mathrm{mi}^{2}\right)\end{array}$} & \multirow{2}{*}{$\begin{array}{l}\text { Main channel } \\
\text { slope }^{2} \text { (ft/mi) }\end{array}$} & \multirow{2}{*}{$\begin{array}{c}\text { Storage (water or } \\
\text { wetlands area) } \\
\text { (percent) }\end{array}$} & \multicolumn{6}{|c|}{ Annual exceedance probability flood-peak streamflows ${ }^{3}$ (ft $\left.{ }^{3} / s\right)$} \\
\hline & & & & & & $10 \%$ & $4 \%$ & $2 \%$ & $1 \%$ & $0.20 \%$ & $1 \%$ plus $^{1}$ \\
\hline \multicolumn{12}{|c|}{ Chippewa Creek } \\
\hline US from Buck Creek & $41^{\circ} 02^{\prime} 54^{\prime \prime}$ & $81^{\circ} 53^{\prime} 58^{\prime \prime}$ & 22.4 & 23.8 & 4.76 & 1,320 & 1,650 & 1,900 & 2,160 & 2,760 & 3,020 \\
\hline US from Westfield Ditch & $41^{\circ} 02^{\prime} 10^{\prime \prime}$ & $81^{\circ} 53^{\prime} 26^{\prime \prime}$ & 26.3 & 20.7 & 4.31 & 1,470 & 1,830 & 2,110 & 2,390 & 3,050 & 3,340 \\
\hline $\begin{array}{l}\text { US from unnamed tributary } \\
\text { (approx } 1,600 \mathrm{ft} \mathrm{US} \\
\text { from Greenwich Rd) }\end{array}$ & $41^{\circ} 01^{\prime} 22^{\prime \prime}$ & $81^{\circ} 52^{\prime} 50^{\prime \prime}$ & 33.9 & 17.7 & 4.25 & 1,720 & 2,140 & 2,460 & 2,780 & 3,540 & 3,880 \\
\hline US from Hubbard Creek & $41^{\circ} 00^{\prime} 38^{\prime \prime}$ & $81^{\circ} 51^{\prime} 52^{\prime \prime}$ & 35.8 & 14.4 & 4.11 & 1,720 & 2,140 & 2,450 & 2,760 & 3,510 & 3,870 \\
\hline US from McCoy Ditch & $40^{\circ} 59^{\prime} 08^{\prime \prime}$ & $81^{\circ} 51^{\prime} 36^{\prime \prime}$ & 46.1 & 11.4 & 3.5 & 2,030 & 2,510 & 2,880 & 3,240 & 4,100 & 4,540 \\
\hline $\begin{array}{l}\text { At USGS streamgage } \\
\quad(03116077)^{4}\end{array}$ & ${ }^{4} 40^{\circ} 57^{\prime} 59^{\prime \prime}$ & ${ }^{4} 81^{\circ} 51^{\prime} 02^{\prime \prime}$ & 50.4 & 9.75 & 3.59 & 2,090 & 2,580 & 2,940 & 3,310 & 4,180 & 4,620 \\
\hline US from Fall Creek & $40^{\circ} 57^{\prime} 25^{\prime \prime}$ & $81^{\circ} 49^{\prime} 21^{\prime \prime}$ & 65.8 & 8.27 & 2.84 & 2,560 & 3,150 & 3,600 & 4,040 & 5,100 & 5,640 \\
\hline US from Tommy Run & $40^{\circ} 57^{\prime} 22^{\prime \prime}$ & $81^{\circ} 48^{\prime} 16^{\prime \prime}$ & 71.4 & 7.37 & 2.72 & 2,670 & 3,280 & 3,740 & 4,200 & 5,300 & 5,880 \\
\hline $\begin{array}{l}\text { US from Little Chippewa } \\
\text { Creek }\end{array}$ & $40^{\circ} 57^{\prime} 48^{\prime \prime}$ & $81^{\circ} 46^{\prime} 43^{\prime \prime}$ & 80.2 & 6.43 & 2.59 & 2,850 & 3,490 & 3,980 & 4,460 & 5,610 & 6,240 \\
\hline US from Styx River & $40^{\circ} 57^{\prime} 32^{\prime \prime}$ & $81^{\circ} 45^{\prime} 56^{\prime \prime}$ & 113 & 6.37 & 2.05 & 3,820 & 4,680 & 5,330 & 5,990 & 7,530 & 8,360 \\
\hline $\begin{array}{l}\text { At USGS streamgage } \\
\quad(03116200)^{4}\end{array}$ & ${ }^{4} 40^{\circ} 56^{\prime} 47^{\prime \prime}$ & ${ }^{4} 81^{\circ} 44^{\prime} 36^{\prime \prime}$ & 146 & 6.07 & 2.41 & 4,490 & 5,470 & 6,210 & 6,950 & 8,700 & 9,700 \\
\hline US from Mill Creek & $40^{\circ} 56^{\prime} 24^{\prime \prime}$ & $81^{\circ} 43^{\prime} 44^{\prime \prime}$ & 147 & 5.31 & 2.42 & 4,380 & 5,320 & 6,030 & 6,750 & 8,420 & 9,420 \\
\hline US from Red Run & $40^{\circ} 56^{\prime} 13^{\prime \prime}$ & $81^{\circ} 43^{\prime} 06^{\prime \prime}$ & 155 & 4.98 & 2.39 & 4,500 & 5,460 & 6,190 & 6,920 & 8,630 & 9,660 \\
\hline US from Silver Creek & $40^{\circ} 55^{\prime} 53^{\prime \prime}$ & $81^{\circ} 40^{\prime} 28^{\prime \prime}$ & 176 & 4.02 & 2.31 & 4,750 & 5,740 & 6,490 & 7,240 & 8,990 & 10,100 \\
\hline At mouth & $40^{\circ} 54^{\prime} 51^{\prime \prime}$ & $81^{\circ} 38^{\prime} 16^{\prime \prime}$ & 188 & 3.16 & 2.48 & 4,690 & 5,630 & 6,340 & 7,050 & 8,720 & 9,840 \\
\hline \multicolumn{12}{|c|}{ Little Chippewa Creek } \\
\hline $\begin{array}{l}\text { US from unnamed tributary } \\
\text { (approx } 1,300 \mathrm{ft} \text { DS } \\
\text { from Steiner } \mathrm{Rd} \text { ) }\end{array}$ & $40^{\circ} 55^{\prime} 55^{\prime \prime}$ & $81^{\circ} 49^{\prime} 33^{\prime \prime}$ & 23.4 & 6.54 & 0.63 & 1,310 & 1,650 & 1,920 & 2,190 & 2,840 & 3,060 \\
\hline $\begin{array}{l}\text { US from unnamed tributary } \\
\text { (approx 3,800 feet US } \\
\text { from S Main St) }\end{array}$ & $40^{\circ} 56^{\prime} 54^{\prime \prime}$ & $81^{\circ} 48^{\prime} 34^{\prime \prime}$ & 26.7 & 6 & 0.57 & 1,430 & 1,800 & 2,090 & 2,380 & 3,090 & 3,320 \\
\hline US from Whitman Ditch & $40^{\circ} 57^{\prime} 19^{\prime \prime}$ & $81^{\circ} 47^{\prime} 28^{\prime \prime}$ & 28.2 & 5.5 & 0.59 & 1,460 & 1,840 & 2,120 & 2,420 & 3,130 & 3,380 \\
\hline At mouth & $40^{\circ} 57^{\prime} 47^{\prime \prime}$ & $81^{\circ} 46^{\prime} 42^{\prime \prime}$ & 32.2 & 6.38 & 0.57 & 1,680 & 2,110 & 2,440 & 2,780 & 3,600 & 3,880 \\
\hline
\end{tabular}


Table 1. Summary of the explanatory variable values used in the regression equations, the initial 10-, 4-, 2-, 1-, and 0.2-percent and 1-percent plus ${ }^{1}$ annual exceedance probability flood-peak streamflow estimates, and locations for the selected streams in Wayne and Medina Counties, Ohio.-Continued

$\left[\mathrm{mi}^{2}\right.$, square mile; ft/mi, foot per mile; $\mathrm{ft}^{3} / \mathrm{s}$, cubic foot per second; \%, percent; US, upstream; ${ }^{\circ}$, degree; ', minute; ", second; approx, approximately; ft, foot; Rd, Road; USGS, U.S. Geological Survey; DS, downstream; S, South; St, Street]

\begin{tabular}{|c|c|c|c|c|c|c|c|c|c|c|c|}
\hline \multirow{2}{*}{ Location description } & \multirow{2}{*}{ Latitude } & \multirow{2}{*}{ Longitude } & \multirow{2}{*}{$\begin{array}{l}\text { Drainage area } \\
\qquad\left(\mathrm{mi}^{2}\right)\end{array}$} & \multirow{2}{*}{$\begin{array}{l}\text { Main channel } \\
\text { slope }^{2}(\mathrm{ft} / \mathrm{mi})\end{array}$} & \multirow{2}{*}{$\begin{array}{l}\text { Storage (water or } \\
\text { wetlands area) } \\
\text { (percent) }\end{array}$} & \multicolumn{6}{|c|}{ Annual exceedance probability flood-peak streamflows ${ }^{3}\left(\mathrm{ft}^{3} / \mathrm{s}\right)$} \\
\hline & & & & & & $10 \%$ & $4 \%$ & $2 \%$ & $1 \%$ & $0.20 \%$ & $1 \%$ plus $^{1}$ \\
\hline \multicolumn{12}{|c|}{ Styx River } \\
\hline $\begin{array}{l}\text { US from unnamed tributary } \\
\text { (approx 1,000 ft DS } \\
\text { from Blake Rd) }\end{array}$ & $41^{\circ} 02^{\prime} 38^{\prime \prime}$ & $81^{\circ} 47^{\prime} 13^{\prime \prime}$ & 6.84 & 67.8 & 3.3 & 715 & 927 & 1,090 & 1,260 & 1,660 & 1,760 \\
\hline $\begin{array}{l}\text { US from unnamed tributary } \\
\text { (approx } 900 \mathrm{ft} \text { US from } \\
\text { Interstate-76) }\end{array}$ & $41^{\circ} 02^{\prime} 01^{\prime \prime}$ & $81^{\circ} 46^{\prime} 43^{\prime \prime}$ & 9.35 & 54 & 2.57 & 895 & 1,160 & 1,360 & 1,570 & 2,070 & 2,190 \\
\hline $\begin{array}{l}\text { US from unnamed tributary } \\
\text { (approx 1,400 ft DS } \\
\text { from Greenwich Rd) }\end{array}$ & $41^{\circ} 01^{\prime} 17^{\prime \prime}$ & $81^{\circ} 46^{\prime} 37^{\prime \prime}$ & 11.3 & 44.4 & 2.73 & 981 & 1,260 & 1,480 & 1,700 & 2,230 & 2,370 \\
\hline $\begin{array}{l}\text { US from unnamed tributary } \\
\text { (approx } 300 \mathrm{ft} \text { US from } \\
\text { Seville Rd) }\end{array}$ & $41^{\circ} 00^{\prime} 39^{\prime \prime}$ & $81^{\circ} 46^{\prime} 13^{\prime \prime}$ & 12.3 & 37.3 & 2.51 & 1,020 & 1,310 & 1,530 & 1,760 & 2,300 & 2,460 \\
\hline US from Holmes Brook & $41^{\circ} 00^{\prime} 22^{\prime \prime}$ & $81^{\circ} 45^{\prime} 59^{\prime \prime}$ & 14.6 & 35.5 & 2.64 & 1,140 & 1,460 & 1,700 & 1,950 & 2,550 & 2,720 \\
\hline $\begin{array}{l}\text { US from unnamed tributary } \\
\text { to Styx River }\end{array}$ & $40^{\circ} 58^{\prime} 38^{\prime \prime}$ & $81^{\circ} 46^{\prime} 19^{\prime \prime}$ & 24.9 & 25.5 & 1.95 & 1,660 & 2,110 & 2,460 & 2,810 & 3,650 & 3,920 \\
\hline At mouth & $40^{\circ} 57^{\prime} 33^{\prime \prime}$ & $81^{\circ} 45^{\prime} 55^{\prime \prime}$ & 29.6 & 23.6 & 2.63 & 1,780 & 2,250 & 2,610 & 2,970 & 3,840 & 4,150 \\
\hline \multicolumn{12}{|c|}{ Unnamed tributary to Styx River } \\
\hline At North Main Street & $40^{\circ} 58^{\prime} 50^{\prime \prime}$ & $81^{\circ} 46^{\prime} 53^{\prime \prime}$ & 2.91 & 70.9 & 0.42 & 470 & 630 & 758 & 892 & 1,220 & 1,250 \\
\hline At mouth & $40^{\circ} 58^{\prime} 37^{\prime \prime}$ & $81^{\circ} 46^{\prime} 20^{\prime \prime}$ & 3.27 & 63.9 & 0.51 & 495 & 662 & 795 & 932 & 1,270 & 1,300 \\
\hline
\end{tabular}

${ }^{1}$ The 1-percent-plus flood elevation is defined as a flood elevation derived using streamflows that are at the upper 84-percent confidence limit as calculated in the gage analysis for the 1-percent annual exceedance probability flood.

${ }^{2}$ The main channel slope (SL10-85) was determined with the 10-85 method described by Koltun (2019).

${ }^{3}$ Annual exceedance probability flood-peak streamflows were determined using the Ohio StreamStats web application that solves regional regression equations (Koltun, 2019).

${ }^{4}$ Locations of U.S. Geological Survey streamgages. 
Table 2. Selected characteristics of U.S. Geological Survey gages within the Chippewa Creek drainage area in Wayne and Medina Counties, Ohio.

[Site locations are shown in figure 1. USGS, U.S. Geological Survey; ft, foot; NAVD 88, North American Vertical Datum of 1988; NGVD 29, National Geodetic Vertical Datum of 1929; Rd, Road; OH, Ohio; ’, degree; ', minute; ", second]

\begin{tabular}{|c|c|c|c|c|c|c|c|}
\hline Site name & $\begin{array}{l}\text { USGS station } \\
\text { number }\end{array}$ & $\begin{array}{c}\text { Drainage } \\
\text { area (square } \\
\text { miles) }\end{array}$ & Latitude & Longitude & $\begin{array}{l}\text { Period of } \\
\text { record }\end{array}$ & $\begin{array}{l}\text { Maximum stage (ft) } \\
\text { (elevation in NAVD } 88 \\
\text { or NGVD } 29 \text { [ft]) }\end{array}$ & $\begin{array}{c}\text { Date of } \\
\text { occurrence }\end{array}$ \\
\hline $\begin{array}{l}\text { Chippewa Creek at } \\
\text { Miller Rd at } \\
\text { Sterling } \mathrm{OH}^{1}\end{array}$ & 03116077 & 50.4 & $40^{\circ} 57^{\prime} 59^{\prime \prime}$ & $81^{\circ} 51^{\prime} 02^{\prime \prime}$ & $2001-21$ & $\begin{array}{l}10.34 \mathrm{ft}(965.28 \mathrm{ft} \\
\text { NAVD 88) }\end{array}$ & June 17, 2019 \\
\hline $\begin{array}{l}\text { Little Chippewa Creek } \\
\text { near Smithville } \mathrm{OH}^{1}\end{array}$ & 03116100 & 16.4 & $40^{\circ} 53^{\prime} 39^{\prime \prime}$ & $81^{\circ} 48^{\prime} 46^{\prime \prime}$ & $1947-72$ & $\begin{array}{l}17.17 \mathrm{ft}(992.17 \mathrm{ft} \\
\text { NGVD 29) }\end{array}$ & July 5, 1969 \\
\hline $\begin{array}{l}\text { Chippewa Creek above } \\
\text { Easton } \mathrm{OH}\end{array}$ & 03116196 & 143 & $40^{\circ} 57^{\prime} 29^{\prime \prime}$ & $81^{\circ} 45^{\prime} 47^{\prime \prime}$ & $2020-21$ & $\begin{array}{l}16.22 \mathrm{ft}(949.90 \mathrm{ft} \\
\text { NAVD 88) }\end{array}$ & March 19, 2020 \\
\hline $\begin{array}{c}\text { Chippewa Creek at } \\
\text { Easton } \mathrm{OH}^{1}\end{array}$ & 03116200 & 146 & $40^{\circ} 56^{\prime} 47^{\prime \prime}$ & $81^{\circ} 44^{\prime} 35^{\prime \prime}$ & $1960-81$ & $\begin{array}{l}18.54 \mathrm{ft}(958.14 \mathrm{ft} \\
\text { NGVD 29) }\end{array}$ & $\begin{array}{l}\text { September 15, } \\
1979\end{array}$ \\
\hline
\end{tabular}

${ }^{1}$ U.S. Geological Survey streamgages used in the hydrologic analyses.

Table 3. Peak-flood streamflow estimates for the 10-, 4-, 2-, 1-, and 0.2-percent annual exceedance probability floods as reported in Koltun (2019), where $R$ is equal to the weighted estimate divided by the regression estimate.

$\left[\mathrm{ft}^{3} / \mathrm{s}\right.$, cubic foot per second; \%, percent; Rd, Road; OH, Ohio]

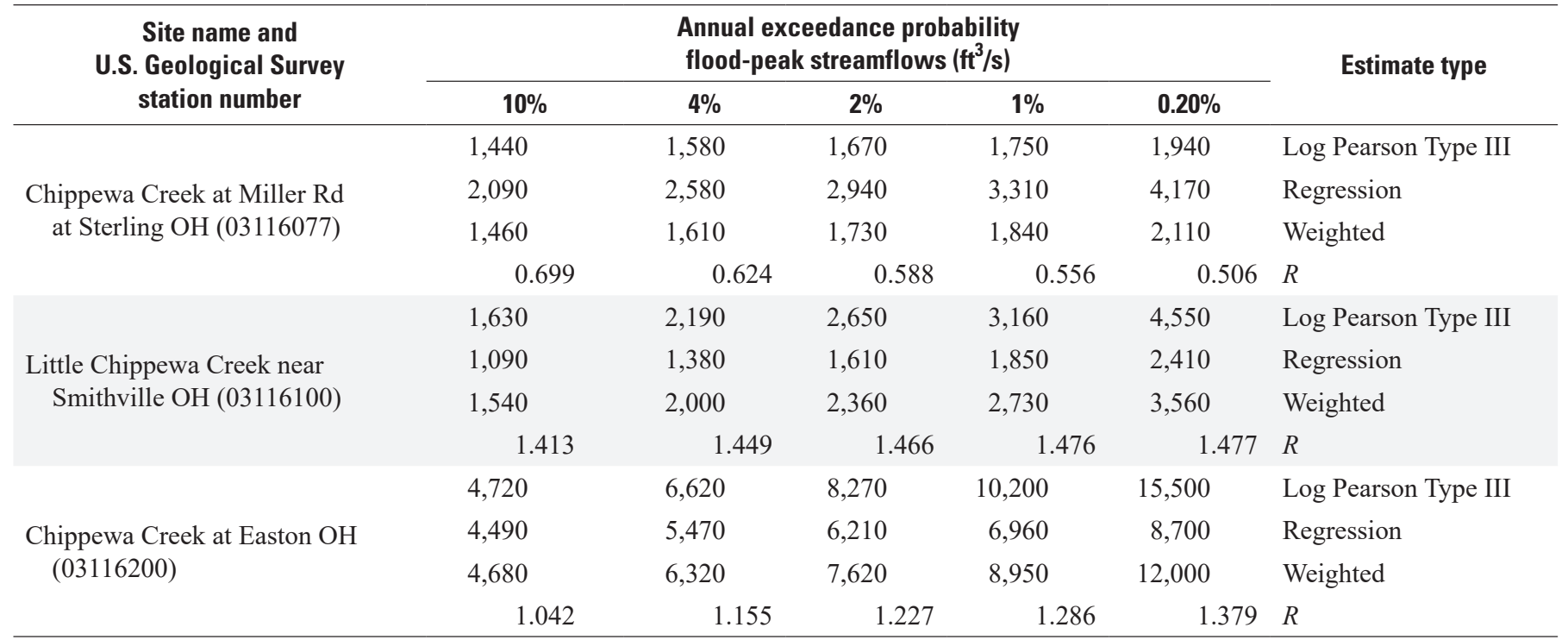


Table 4. Summary of the final 10-, 4-, 2-, 1-, and 0.2-percent and 1-percent plus annual exceedance probability flood-peak streamflow estimates and locations for the selected streams in Wayne and Medina Counties, Ohio.

$\left[\mathrm{ft}^{3} / \mathrm{s}\right.$, cubic foot per second; \%, percent; US, upstream; approx, approximately; ft, foot; Rd, Road; USGS, U.S. Geological Survey; DS, downstream; S, South; St, Street]

\begin{tabular}{|c|c|c|c|c|c|c|}
\hline \multirow{2}{*}{ Location description } & \multicolumn{6}{|c|}{ Annual exceedance probability flood-peak streamflows ${ }^{1}\left(\mathrm{ft}^{3} / \mathrm{s}\right)$} \\
\hline & $10 \%$ & $4 \%$ & $2 \%$ & $1 \%$ & $0.20 \%$ & $1 \%$ plus $^{2}$ \\
\hline \multicolumn{7}{|c|}{ Chippewa Creek } \\
\hline US from Westfield Ditch & 950 & 1,060 & 1,150 & 1,230 & 1,430 & 1,720 \\
\hline $\begin{array}{l}\text { US from unnamed tributary (approx 1,600 ft US } \\
\text { from Greenwich Rd) }\end{array}$ & 1,120 & 1,250 & 1,350 & 1,440 & 1,670 & 2,010 \\
\hline US from McCoy Ditch & 1,380 & 1,520 & 1,640 & 1,740 & 2,010 & 2,430 \\
\hline At USGS streamgage $(03116077)^{3}$ & ${ }^{3} 1,460$ & ${ }^{3} 1,610$ & ${ }^{3} 1,730$ & ${ }^{3} 1,840$ & ${ }^{3} 2,120$ & ${ }^{3} 2,570$ \\
\hline US from Fall Creek & 2,260 & 2,690 & 3,020 & 3,340 & 4,120 & 4,660 \\
\hline US from Tommy Run & 2,540 & 3,070 & 3,480 & 3,890 & 4,860 & 5,430 \\
\hline US from Mill Creek & 4,700 & 6,350 & 7,660 & 8,980 & 12,100 & 12,600 \\
\hline US from Red Run & 4,860 & 6,570 & 7,920 & 9,270 & 12,400 & 13,000 \\
\hline US from Silver Creek & 5,290 & 7,130 & 8,580 & 10,000 & 13,400 & 14,000 \\
\hline At mouth & 5,530 & 7,430 & 8,930 & 10,500 & 13,900 & 14,600 \\
\hline \multicolumn{7}{|c|}{ Little Chippewa Creek } \\
\hline $\begin{array}{l}\text { US from unnamed tributary (approx 1,300 ft DS } \\
\text { from Steiner Rd) }\end{array}$ & 1,380 & 1,750 & 2,040 & 2,320 & 3,010 & 3,240 \\
\hline $\begin{array}{l}\text { US from unnamed tributary (approx 3,800 ft US } \\
\text { from S Main St) }\end{array}$ & 1,430 & 1,800 & 2,090 & 2,380 & 3,090 & 3,320 \\
\hline $\begin{array}{l}\text { US from unnamed tributary (approx 1,400 ft DS } \\
\text { from Greenwich Rd) }\end{array}$ & 981 & 1,260 & 1,480 & 1,700 & 2,230 & 2,370 \\
\hline $\begin{array}{l}\text { US from unnamed tributary (approx } 300 \mathrm{ft} \mathrm{US} \\
\text { from Seville Rd) }\end{array}$ & 1,020 & 1,310 & 1,530 & 1,760 & 2,300 & 2,460 \\
\hline US from Holmes Brook & 1,140 & 1,460 & 1,700 & 1,950 & 2,550 & 2,720 \\
\hline US from unnamed tributary to Styx River & 1,660 & 2,110 & 2,460 & 2,810 & 3,650 & 3,920 \\
\hline At mouth & 1,780 & 2,250 & 2,610 & 2,970 & 3,840 & 4,150 \\
\hline \multicolumn{7}{|c|}{ Unnamed tributary to Styx River } \\
\hline At North Main Street & 470 & 630 & 758 & 892 & 1,220 & 1,250 \\
\hline At mouth & 495 & 662 & 795 & 932 & 1,270 & 1,300 \\
\hline
\end{tabular}

\footnotetext{
${ }^{1}$ Annual exceedance probability flood-peak streamflows were initially estimated using the Ohio StreamStats web application (Koltun, 2019). Where applicable, the estimates were gage-weighted using techniques in Koltun (2019) or adjusted by multiplying by a ratio equal to the regression estimate for the ungaged location divided by the regression estimate for the nearest gage location, both computed using drainage-area only equations in Koltun (2019).

${ }^{2}$ The 1-percent-plus flood elevation is defined as a flood elevation derived using streamflows that are at the upper 84-percent confidence limit as calculated in the gage analysis for the 1- percent annual exceedance probability flood.

${ }^{3}$ Locations of U.S. Geological Survey streamgages.
} 
Table 5. Survey characteristics and hydraulic parameters used in the hydraulic models.

[DEM, digital elevation model; ft/ft, foot per foot]

\begin{tabular}{|c|c|c|c|c|c|c|c|c|c|c|}
\hline \multirow{3}{*}{ Stream name } & \multirow{3}{*}{$\begin{array}{l}\text { Base line } \\
\text { reference } \\
\text { location }\end{array}$} & \multirow{3}{*}{$\begin{array}{l}\text { Study } \\
\text { reach } \\
\text { length } \\
\text { (miles) }\end{array}$} & \multirow{3}{*}{$\begin{array}{c}\text { Number of } \\
\text { surveyed } \\
\text { cross } \\
\text { sections }\end{array}$} & \multirow{3}{*}{$\begin{array}{l}\text { Number of } \\
\text { cross } \\
\text { sections } \\
\text { derived } \\
\text { from DEM }\end{array}$} & \multirow{3}{*}{$\begin{array}{l}\text { Number of } \\
\text { hydraulic } \\
\text { structures }\end{array}$} & \multirow{3}{*}{$\begin{array}{l}\text { Slope used } \\
\text { for normal } \\
\text { depth }^{2} \\
\text { calculation } \\
\text { (tt/ft) }\end{array}$} & \multicolumn{4}{|c|}{ Manning's roughness coefficient $(n)$} \\
\hline & & & & & & & \multicolumn{2}{|c|}{ Main channel } & \multicolumn{2}{|c|}{ Overbanks } \\
\hline & & & & & & & $\begin{array}{l}\text { Lowest } \\
\text { value }\end{array}$ & $\begin{array}{l}\text { Highest } \\
\text { value }\end{array}$ & $\begin{array}{l}\text { Lowest } \\
\text { value }\end{array}$ & $\begin{array}{l}\text { Highest } \\
\text { value }\end{array}$ \\
\hline Chippewa Creek & Mouth & 23.5 & 132 & 434 & 32 & 0.00034 & 0.024 & 9 & 0.049 & 0.085 \\
\hline $\begin{array}{l}\text { Little Chippewa } \\
\text { Creek }\end{array}$ & Mouth & 3.4 & 15 & 70 & 3 & 0.00220 & 0.036 & 0.038 & 0.036 & 0.065 \\
\hline Styx River & Mouth & 8.1 & 79 & 202 & 19 & 0.00130 & 0.03 & 0.04 & 0.028 & 0.075 \\
\hline $\begin{array}{l}\text { Unnamed tributary } \\
\text { to Styx River }\end{array}$ & Mouth & 1.2 & 18 & 63 & 4 & 0.00150 & 0.038 & 0.046 & 0.028 & 0.052 \\
\hline
\end{tabular}

${ }^{1}$ Location from which the river stationing is measured upstream, in feet.

${ }^{2}$ Normal depth is the depth when the streamflow is uniform, steady, one-dimensional, and is not affected by downstream obstructions. Flow is considered uniform if the energy line, water surface, and channel bottom all are parallel (Chow, 1959).

system at 15 benchmark locations had a root-mean-square error of 0.06 foot (ft) when compared with National Geodetic Survey published elevations.

The USGS field crews surveyed a total of 244 channel cross sections and 58 hydraulic structures (table 5). The cross sections were surveyed to provide ground elevations below stream water surfaces that are not provided by conventional light detection and ranging (lidar). The structures were surveyed for geometrical data that have the potential to affect water-surface elevations during floods along the streams.

\section{Topographic and Bathymetric Data}

Cross-section elevation data were obtained from a digital elevation model (DEM) that was derived from lidar data collected during March 2006 for the Ohio Geographically Referenced Information Program (Ohio Geographically Referenced Information Program, 2007). The lidar data have a horizontal resolution that meets National Map Accuracy Standards with a vertical accuracy of plus or minus $1.0 \mathrm{ft}$ at a 95-percent confidence level for the "open terrain" land-cover category (root-mean-square error of $0.5 \mathrm{ft}$ ) (Federal Geographic Data Committee, 1998). By this criterion, the lidar data support production of 2-ft contours (Dewberry, 2012) and a DEM that has a vertical accuracy of plus or minus $1.0 \mathrm{ft}$. The DEM data from the Ohio Geographically Referenced Information Program (2007) were used to develop 2-ft contours by USGS personnel.

HEC-GeoRAS was used to extract elevation data from the DEM for a total of 769 cross sections (table 5) for use in the HEC-RAS models. For all modeled stream reaches, the maximum distance between cross sections (conventionally surveyed and DEM derived) was less than or equal to $500 \mathrm{ft}$. Spacing between cross sections averaged $780 \mathrm{ft}$ for conventionally surveyed cross sections and $250 \mathrm{ft}$ for DEM-derived cross sections. DEM-derived cross sections were collocated with the locations of the in-channel field-surveyed cross sections where available. In those cases, DEM-based elevations within the channel were substituted with in-channel survey elevations to form composite cross sections. In-channel elevations for DEM-derived cross sections that did not have surveyed channels were estimated by interpolating between the closest field-surveyed cross sections as a function of distance along the hydraulic baseline.

\section{Hydraulic Modeling}

The hydraulic base lines for all modeled stream reaches were referenced to feet upstream from their respective mouths (table 5). Normal depth boundary conditions (table 5) were used for all streams. Normal depth is defined as the depth when the streamflow is uniform, steady, one-dimensional, and is not affected by downstream obstructions. Streamflow is considered uniform if the energy line, water surface, and channel bottom all are parallel (Chow, 1959). The friction slopes (equal to the channel slopes) for the normal depth calculations were determined from field surveys done near the downstream limits. The HEC-RAS models were used to generate seven water-surface profiles corresponding to the 10-, 4-, 2-, 1-, and 0.2 -percent and 1-percent plus AEP floods and a regulatory floodway. A "regulatory floodway" is defined as the channel of a river or other watercourse and the adjacent land areas that 
must be reserved to discharge the 1-percent AEP flood without increasing the water-surface elevation more than a designated height (Federal Emergency Management Agency, 2020).

\section{Overflow Reaches}

During the modeling process, it was noted that the main channels of Chippewa Creek and the unnamed tributary to Styx River did not contain all the modeled streamflows. As a result, the hydraulic models for Chippewa Creek and the unnamed tributary to Styx River include overflow channels. The overflow channels were used to model streamflow that spills out of the main channel, travels for a distance, and then rejoins the main channel at a downstream location. The overflow channels were connected to the main channels using junctions within HEC-RAS. Junctions are defined as locations where two or more streams join together or split apart (U.S. Army Corps of Engineers, 2016).

For Chippewa Creek, streamflows were contained within the main channel for 10- and 4- percent AEP flood-peak streamflows. However, for the higher streamflows, water spills out of the main channel from the right overbank near Gloria Glens Park (fig. 2) and flowed through a swampy wooded area before entering a small tributary (Westfield Ditch) and returning to the main channel of Chippewa Creek just upstream from Interstate 71. In the hydraulic model, Chippewa Creek was split into an upper, middle, and lower reach at the split and join junctions. Streamflows routed along the overflow reach were subtracted from the streamflows routed down the Chippewa Creek middle reach. The model was run iteratively until the water-surface elevations for the overflow reach and the upstream end of the Chippewa Creek middle reach at the split junction were equal. For the floodway run, the streamflow was contained within the main channel. The final balanced streamflows are listed in table 6.

The unnamed tributary to Styx River also has split flow. Upstream from North Main Street (fig. 3), there are two points or junctions (split A and split B) where the streamflow spills out of the main channel before rejoining just upstream from North Main Street. Overflow at the split B junction leaves the main channel of the unnamed tributary to Styx River and moves parallel before joining with additional overflow near the split A junction (fig. 3). The combined overflow then continues downgradient, eventually rejoining the main stem just upstream from where the unnamed tributary to Styx River crosses under North Main Street. Streamflows routed along the overflow reaches were subtracted from the streamflows routed down the unnamed tributary to Styx River reaches B and C. The unnamed tributary to Styx River model was run iteratively until (1) the water-surface elevations for overflow 2 reach and the unnamed tributary to Styx River reach C at the split B junction were balanced and (2) streamflow no longer escaped from the unnamed tributary to Styx River reach B to the overflow reach A downstream from the split A junction.

The maximum capacity of the unnamed tributary to Styx River reach B is 446 cubic feet per second. Any increase in streamflow greater than 446 cubic feet per second results in streamflow overflowing the main channel at multiple locations along the east overbank and moving into the overflow reach A. There was no overflow into the overflow 2 reach for the 10 and 4- percent AEP flood-peak streamflows. For the floodway run, all streamflow was contained within the main channel until split A junction, after which 413 cubic feet per second of streamflow escaped as overflow. The final balanced streamflows are listed in table 7.

\section{Development of Flood-Inundation Boundaries}

Flood-inundation boundaries were mapped with a geographic information system for three water-surface profiles (1- and 0.2-percent AEP floods and a regulatory floodway) using flood-profile data, the DEM, and contours developed from the DEM. Initial flood-inundation boundaries were developed for each profile using the HEC-GeoRAS software (U.S. Army Corps of Engineers, 2009). HEC-GeoRAS provides a set of procedures, tools, and utilities for processing HEC-RAS outputs and geospatial data in ArcGIS. Floodinundation boundaries for the inundated areas were refined in ArcMap (Environmental Systems Research Institute, 2020) using the DEM-derived 2-ft contours to ensure hydraulically reasonable boundary transitions between model cross sections.

Any inundated areas that were disconnected from the main channel were examined to determine whether there were artificial connections with the main river, such as through culverts under roadways. Where such connections existed, the mapped inundated areas were retained in their respective flood-inundation boundaries; otherwise, the disconnected inundated areas were deleted. 
$81^{\circ} 52^{\prime} 0^{\prime \prime}$

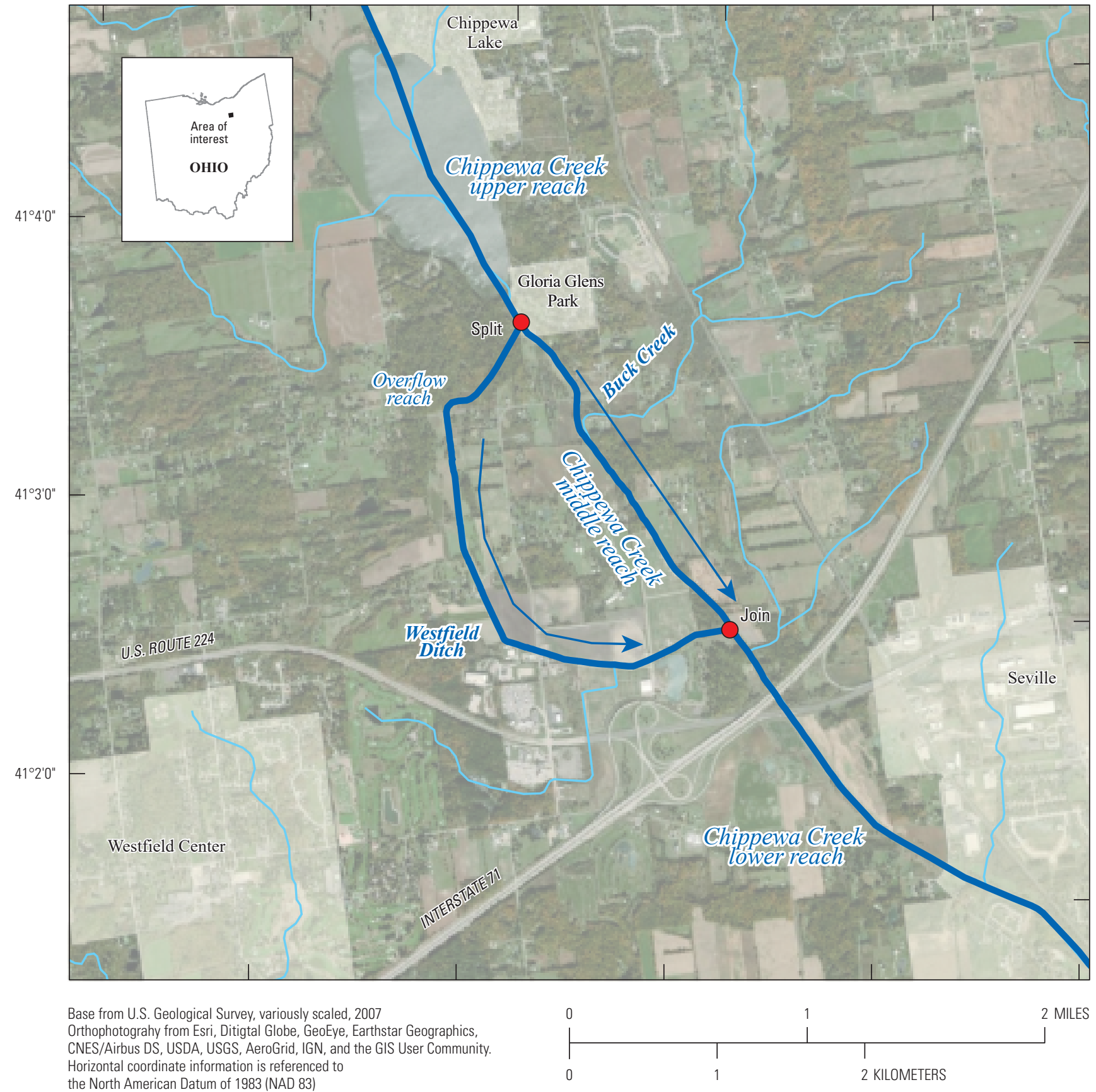

the North American Datum of 1983 (NAD 83)

\section{EXPLANATION}

\begin{tabular}{|lll}
\hline Corporate limits & \multicolumn{1}{l}{ Flow arrow, indicates direction of water flow } \\
Study reaches & & Streams \\
& & Streamflow junctions
\end{tabular}

Figure 2. Locations of flow path and junctions at which flow leaves and returns to the main channel of Chippewa Creek. 


\section{Hydrologic and Hydraulic Analyses of Selected Streams near the City of Rittman}

Table 6. Streamflows resulting in balanced water-surface elevations between the main stem of Chippewa Creek and the overflow reach or Westfield Ditch.

$\left[\mathrm{ft}^{3} / \mathrm{s}\right.$, cubic foot per second; \%, percent; US, upstream; DS, downstream; N/A, not applicable]

\begin{tabular}{|c|c|c|c|c|c|c|c|c|c|}
\hline \multirow{2}{*}{ Stream } & \multirow{2}{*}{ Reach } & \multirow{2}{*}{ Location } & \multicolumn{7}{|c|}{ Annual exceedance probability flood-peak streamflows $\left(\mathrm{ft}^{3} / \mathrm{s}\right)$} \\
\hline & & & $10 \%$ & $4 \%$ & $2 \%$ & $1 \%$ & $0.20 \%$ & $1 \%$ plus & Floodway \\
\hline \multirow{3}{*}{ Chippewa Creek } & Upper & US from split & 854 & 957 & 1,040 & 1,110 & 1,300 & 1,560 & 1,110 \\
\hline & Middle & DS from Buck Creek & 950 & 1,060 & 1,098 & 1,128 & 1,192 & 1,267 & 1,230 \\
\hline & Lower & $\begin{array}{l}\text { DS from Westfield } \\
\text { Ditch }\end{array}$ & 1,120 & 1,250 & 1,350 & 1,440 & 1,670 & 2,010 & 1,440 \\
\hline
\end{tabular}

Table 7. Streamflows resulting in balanced water-surface elevations between the main stem of the unnamed tributary to Styx River and the overflow reaches.

$\left[\mathrm{ft}^{3} / \mathrm{s}\right.$, cubic foot per second; \%, percent; US, upstream; DS, downstream; N/A, not applicable]

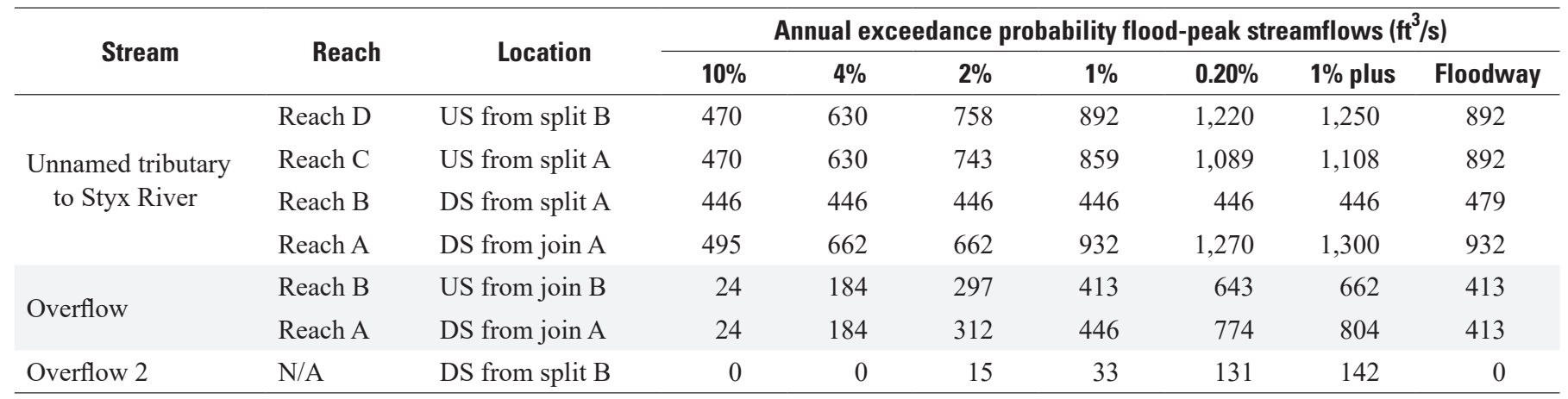




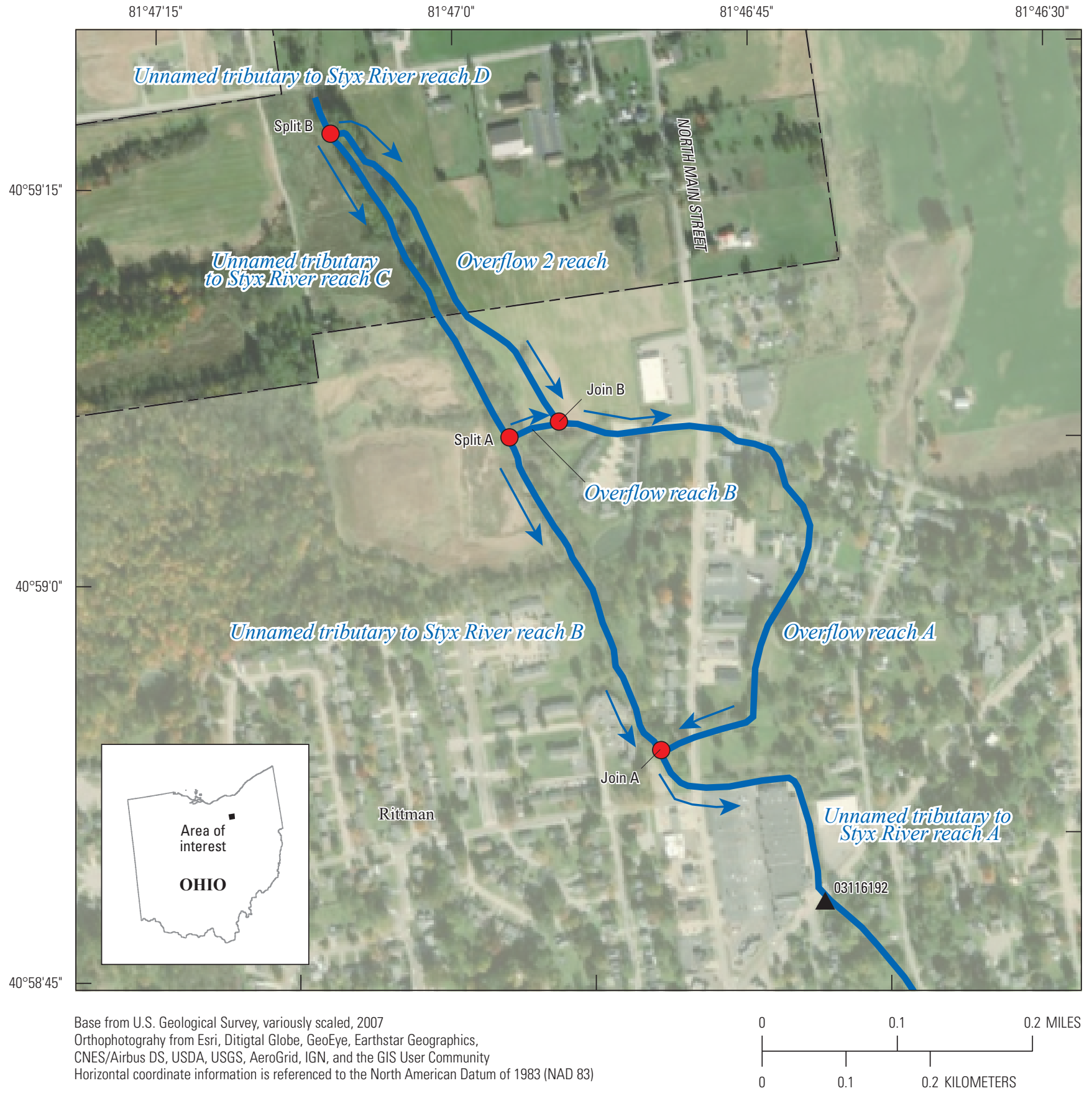

\section{EXPLANATION}

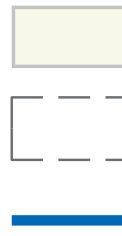

Corporate areas

$\longrightarrow$ Flow arrow, indicates direction of water flow

Corporate boundaries

03116192

U.S. Geological Survey streamgage and number

Study reaches

Streamflow junctions

Figure 3. Locations of flow paths and points at which flow leaves and returns to the main channel of the unnamed tributary to Styx River. 


\section{Data Dissemination}

All data used in the creation of the flood-inundation boundaries are available through a USGS data release at https://doi.org/10.5066/P9W6ROMC (Ostheimer, 2021) and will be submitted to FEMA for inclusion in updated FISs for Wayne and Medina Counties. FEMA has the sole statutory responsibility for publishing an FIS and regulatory flood-plain boundaries. As a result, the information in the data release should not be used for regulatory purposes until they have been published by FEMA in an FIS. Floodway data tables, listing floodway characteristics and 1-percent AEP flood and floodway water-surface elevations at model cross sections on each stream, are presented in appendix 1.

\section{Summary}

The U.S. Geological Survey, in cooperation with the city of Rittman, Ohio, and the Muskingum Watershed Conservancy District, did a study to provide data with which to update and expand parts of two Federal Emergency Management Agency Flood Insurance Studies. The study covered a total of 36.2 miles on four streams (Chippewa Creek, Little Chippewa Creek, Styx River, and the unnamed tributary to Styx River) in the Chippewa Creek Basin. Water-surface profiles were developed for the 10-, 4-, 2-, 1-, and 0.2-percent and 1-percent plus annual exceedance probability floods and a regulatory floodway. Flood boundaries for each stream were mapped for the 1- and 0.2-percent annual exceedance probability floods and a regulatory floodway. The water-surface profiles and flood-inundation boundaries can help emergency planners and the public make more informed decisions about flood risk.

The U.S. Army Corps of Engineers Hydrologic Engineering Center's River Analysis and Geo River Analysis Systems were used to compute water-surface profiles and help delineate estimated flood-inundation-area boundaries. Model input included cross sections derived from a digital elevation model supplemented with field surveys of open-channel cross sections and hydraulic structures, estimates of roughness values, downstream boundary conditions, and annual exceedance probability flood estimates from regional regression equations and historical streamflow data. All data used in the creation of the flood-inundation boundaries are available through a U.S. Geological Survey data release (Ostheimer, 2021) and will be submitted to the Federal Emergency Management Agency for inclusion in updated Flood Insurance studies for Wayne and Medina Counties.

\section{References Cited}

Chow, V.T., 1959, Open-channel hydraulics: New York, McGraw Hill, 680 p.

Dewberry, 2012, National Enhanced Elevation AssessmentFinal report: Fairfax, Va., 84 p., accessed March 5, 2020, at https://www.dewberry.com/services/geospatial/nationalenhanced-elevation-assessment.

Environmental Systems Research Institute, 2020, ArcGISDiscover your power with ArcGIS: Environmental Systems Research Institute web page, accessed March 5, 2020, at https://www.esri.com/en-us/arcgis/about-arcgis/overview.

Federal Emergency Management Agency, 2009, Flood Insurance Study, Wayne County, Ohio, and incorporated areas: Washington D.C., Flood Insurance Study Number 39169CV00A, 66 p., accessed March 5, 2020, at https://msc.fema.gov/data/39/S/PDF/39169CV000A.pdf.

Federal Emergency Management Agency, 2013, Flood Insurance Study, Medina County, Ohio, and incorporated areas: Washington D.C., Flood Insurance Study Number 39103CV001B, 98 p., accessed March 5, 2020, at https://msc.fema.gov/data/39/S/PDF/39103CV001B.pdf.

Federal Emergency Management Agency, 2019, Guidance for flood risk analysis and mapping: Washington D.C., 20 p. accessed March 5, 2020, at https://www.fema.gov/sites/ default/files/2020-02/General_Hydrologic_Considerations_ Guidance_Feb_2019.pdf.

Federal Emergency Management Agency, 2020, Floodway: Federal Emergency Management Agency web page, accessed March 5, 2020, at https:/www.fema.gov/glossary/ floodway.

Federal Geodetic Control Committee, 1984, Standards and specifications for geodetic control networks: Rockville, Md., Federal Geodetic Control Committee, accessed March 5, 2020, at https:/www.ngs.noaa.gov/FGCS/tech_ pub/1984-stds-specs-geodetic-control-networks.pdf.

Federal Geographic Data Committee, 1998, Geospatial positioning accuracy standards part 3-Nation standard for spatial data accuracy: Reston, Va., U.S. Geological Survey, FGDC-STD-007.3-1998, accessed March 5, 2020, at https://www.fgdc.gov/standards/projects/FGDC-standardsprojects/accuracy/part3/chapter3/. 
Interagency Advisory Committee on Water Data, 1982, Guidelines for determining flood flow frequency: Reston, Va., U.S. Geological Survey, Bulletin 17B of the Hydrology Subcommittee, Office of Water Data Coordination, 183 p.

Koltun, G.F., 2019, Flood-frequency estimates for Ohio streamgages based on data through water year 2015 and techniques for estimating flood-frequency characteristics of rural, unregulated Ohio streams: U.S. Geological Survey Scientific Investigations Report 2019-5018, 25 p., accessed March 5, 2020, at https://doi.org/10.3133/sir20195018.

Ohio Geographically Referenced Information Program, 2007, OSIP data downloads, accessed March 5, 2020, at https://ogrip.oit.ohio.gov/ProjectsInitiatives/OSIPDataDown loads.aspx.

Ostheimer, C.J., 2021, Geospatial datasets and hydraulic models for selected streams near Rittman in Wayne and Medina Counties, Ohio: U.S. Geological Survey data release, https://doi.org/10.5066/P9W6ROMC.

PRISM Climate Group, 2012, 30-year normals (800m): PRISM Climate Group, Oregon State University, accessed March 5, 2020, at https://prism.oregonstate.edu/normals/.

Rydlund, P.H., Jr., and Densmore, B.K., 2012, Methods of practice and guidelines for using survey-grade global navigation satellite systems (GNSS) to establish vertical datum in the United States Geological Survey: U.S. Geological Survey Techniques and Methods, book 11, chap. D1, 102 p. with appendixes.

U.S. Army Corps of Engineers, 2009, HEC-GeoRAS-GIS tools for support of HEC-RAS using ArcGIS-User's manual, version 4.2: Davis, Calif., U.S. Army Corps of Engineers Institute for Water Resources, Hydrologic Engineering Center [variously paged], accessed March 5, 2020, at https://www.hec.usace.army.mil/software/hecgeoras/documentation/HEC-GeoRAS42_UsersManual.pdf.

U.S. Army Corps of Engineers, 2016, HEC-RAS - River analysis system-User's manual, version 5.0: Davis, Calif., U.S. Army Corps of Engineers Institute for Water Resources, Hydrologic Engineering Center, [variously paged], accessed March 5, 2020, at https://www.hec.usace .army.mil/software/hec-ras/documentation/HEC-RAS $\% 20$ 5.0\%20Users\%20Manual.pdf.
U.S. Census Bureau, 2010a, Quick facts-Wayne County, Ohio: United States Census Bureau web page, accessed March 5, 2020, at https:/www.census.gov/quickfacts/fact/ table/waynecountyohio,US/PST045219.

U.S. Census Bureau, 2010b, Quick facts-Medina County, Ohio: United States Census Bureau web page, accessed March 5, 2020, at https://www.census.gov/quickfacts/fact/ table/medinacountyohio,US/PST045219.

U.S. Census Bureau, 2010c, Quick facts-Rittman city, Ohio: United States Census Bureau web page, accessed March 5, 2020, at https://www.census.gov/quickfacts/fact/table/ rittmancityohio,US/PST045219.

U.S. Geological Survey, 2014, Data-Land cover: Sioux Fall, S. Dak., U.S. Geological Survey metadata, accessed March 5, 2020, at https://www.mrlc.gov/data?f\%5B0\%5D= category $\% 3$ Aland $\% 20$ cover\&f $\% 5 \mathrm{~B} 1 \% 5 \mathrm{D}=$ regi on $\% 3$ Aconus\&f $\% 5 B 2 \% 5 \mathrm{D}=$ year $\% 3 \mathrm{~A} 2011$.

U.S. Geological Survey, 2020a, USGS 03116077 Chippewa Creek at Miller Rd at Sterling OH: U.S. Geological Survey National Water Information System database, accessed March 5, 2020, at https://dx.doi.org/10.5066/F7P55KJN. [Site information directly accessible at https://waterdata .usgs.gov/oh/nwis/inventory/?site_no $=03116077$.

U.S. Geological Survey, 2020b, USGS 03116192 Unnamed Tributary to Styx River at Rittman OH: U.S. Geological Survey National Water Information System database, accessed March 5, 2020, at https://doi.org/10.5066/F7P5 $5 \mathrm{KJN}$. [Site information directly accessible at https:/ /waterdata.usgs.gov/oh/nwis/inventory/?site_no=03116192.]

U.S. Geological Survey, 2020c, USGS 03116200 Chippewa Creek at Easton OH: U.S. Geological Survey National Water Information System database, accessed March 5, 2020, at https://doi.org/10.5066/F7P55KJN. [Site information directly accessible at https://waterdata.usgs.gov/oh/ nwis/inventory/?site_no $=03116200$.]

U.S. Geological Survey, 2020d, USGS water data for the Nation: U.S. Geological Survey National Water Information System database, accessed March 5, 2020, at https://doi.org/ 10.5066/F7P55KJN. 



\section{Appendix 1}

Table 1.1. Floodway data table for Chippewa Creek, Wayne and Medina Counties, Ohio.

Table 1.2. Floodway data table for Little Chippewa Creek, Wayne County, Ohio.

Table 1.3. Floodway data table for Styx River, Wayne and Medina Counties, Ohio.

Table 1.4. Floodway data table for unnamed tributary to Styx River, Wayne County, Ohio.

Table 1.5. Floodway data table for unnamed tributary to Styx River overflow, Wayne County, Ohio. 
Table 1.1. Floodway data table for Chippewa Creek, Wayne and Medina Counties, Ohio.

[\%, percent; AEP, annual exceedance probability; BW, backwater; NAVD 88, North American Vertical Datum of 1988]

\begin{tabular}{|c|c|c|c|c|c|c|c|c|}
\hline \multicolumn{2}{|c|}{ Location } & \multicolumn{3}{|c|}{ Floodway ${ }^{1}$} & \multicolumn{4}{|c|}{$1 \%$ AEP water-surface elevation } \\
\hline $\begin{array}{l}\text { Cross } \\
\text { section } \\
\text { letter }\end{array}$ & $\begin{array}{c}\text { Station }^{2} \\
\text { (feet) }\end{array}$ & $\begin{array}{l}\text { Width } \\
\text { (feet) }\end{array}$ & $\begin{array}{c}\text { Cross } \\
\text { sectional } \\
\text { area } \\
\text { (square feet) }\end{array}$ & $\begin{array}{c}\text { Mean } \\
\text { velocity } \\
\text { (feet per } \\
\text { second) }\end{array}$ & $\begin{array}{l}\text { Modeled stream with } \\
\text { BW (if any) from } \\
\text { receiving stream } \\
\text { (feet, NAVD 88) }\end{array}$ & $\begin{array}{l}\text { Modeled stream } \\
\text { without BW from } \\
\text { receiving stream } \\
\text { (feet, NAVD 88) }\end{array}$ & $\begin{array}{l}\text { Modeled stream with } \\
\text { floodway without BW } \\
\text { from receiving stream } \\
\text { (feet, NAVD 88) }\end{array}$ & $\begin{array}{c}\text { Increase in water surface } \\
\text { (without BW) } \\
\text { due to } \\
\text { floodway (feet) }\end{array}$ \\
\hline $\mathrm{A}$ & 369 & 635 & 4,398 & 2.4 & 946.7 & 946.7 & 947.6 & 0.9 \\
\hline B & 751 & 167 & 1,839 & 5.7 & 946.8 & 946.8 & 947.6 & 0.8 \\
\hline $\mathrm{C}$ & 777 & 172 & 2,036 & 5.2 & 947.7 & 947.7 & 948.7 & 1.0 \\
\hline $\mathrm{D}$ & 1,288 & 180 & 2,258 & 4.6 & 948.2 & 948.2 & 949.1 & 0.9 \\
\hline $\mathrm{E}$ & 1,768 & 120 & 1,546 & 6.8 & 948.3 & 948.3 & 949.2 & 0.9 \\
\hline $\mathrm{F}$ & 1,797 & 135 & 1,773 & 5.9 & 948.7 & 948.7 & 949.6 & 0.9 \\
\hline G & 1,848 & 135 & 1,846 & 5.7 & 949.8 & 949.8 & 950.2 & 0.4 \\
\hline $\mathrm{H}$ & 1,893 & 185 & 2,231 & 4.7 & 950.1 & 950.1 & 950.4 & 0.3 \\
\hline I & 2,372 & 350 & 3,534 & 3.0 & 950.5 & 950.5 & 950.7 & 0.2 \\
\hline $\mathrm{J}$ & 3,970 & 700 & 7,038 & 1.5 & 950.7 & 950.7 & 951.1 & 0.4 \\
\hline K & 4,896 & 146 & 1,731 & 6.1 & 950.6 & 950.6 & 951.0 & 0.4 \\
\hline $\mathrm{L}$ & 5,019 & 148 & 1,760 & 6.0 & 950.8 & 950.8 & 951.2 & 0.4 \\
\hline $\mathrm{M}$ & 5,402 & 300 & 3,693 & 2.8 & 951.7 & 951.7 & 952.0 & 0.3 \\
\hline $\mathrm{N}$ & 5,582 & 400 & 4,927 & 2.2 & 951.8 & 951.8 & 952.1 & 0.3 \\
\hline $\mathrm{O}$ & 7,812 & 950 & 5,934 & 1.8 & 951.9 & 951.9 & 952.3 & 0.4 \\
\hline $\mathrm{P}$ & 7,851 & 918 & 6,401 & 1.6 & 952.4 & 952.4 & 952.8 & 0.4 \\
\hline Q & 9,763 & 1,450 & 15,059 & 0.7 & 952.5 & 952.5 & 953.1 & 0.6 \\
\hline $\mathrm{R}$ & 17,055 & 1,600 & 10,648 & 0.9 & 952.7 & 952.7 & 953.3 & 0.6 \\
\hline $\mathrm{S}$ & 17,096 & 1,600 & 10,828 & 0.9 & 952.7 & 952.7 & 953.3 & 0.6 \\
\hline $\mathrm{T}$ & 22,936 & 1,504 & 11,182 & 0.9 & 952.9 & 952.9 & 953.5 & 0.6 \\
\hline $\mathrm{U}$ & 22,976 & 1,504 & 10,968 & 0.9 & 952.9 & 952.9 & 953.5 & 0.6 \\
\hline $\mathrm{V}$ & 25,238 & 2,250 & 12,288 & 0.8 & 953.0 & 953.0 & 953.6 & 0.6 \\
\hline W & 28,346 & 2,719 & 17,406 & 0.5 & 953.0 & 953.0 & 953.7 & 0.7 \\
\hline $\mathrm{X}$ & 28,391 & 2,719 & 17,049 & 0.5 & 953.0 & 953.0 & 953.7 & 0.7 \\
\hline $\mathrm{Y}$ & 28,862 & 2,800 & 17,209 & 0.5 & 953.0 & 953.0 & 953.7 & 0.7 \\
\hline $\mathrm{Z}$ & 31,506 & 1,200 & 7,312 & 1.2 & 953.1 & 953.1 & 953.8 & 0.7 \\
\hline $\mathrm{AA}$ & 33,360 & 590 & 3,469 & 2.6 & 953.3 & 953.3 & 954.1 & 0.8 \\
\hline $\mathrm{AB}$ & 34,277 & 153 & 1,911 & 4.7 & 953.5 & 953.5 & 954.3 & 0.8 \\
\hline
\end{tabular}


Table 1.1. Floodway data table for Chippewa Creek, Wayne and Medina Counties, Ohio.-Continued

[\%, percent; AEP, annual exceedance probability; BW, backwater; NAVD 88, North American Vertical Datum of 1988]

\begin{tabular}{|c|c|c|c|c|c|c|c|c|}
\hline \multicolumn{2}{|c|}{ Location } & \multicolumn{3}{|c|}{ Floodway $^{1}$} & \multicolumn{4}{|c|}{ 1\% AEP water-surface elevation } \\
\hline $\begin{array}{l}\text { Cross } \\
\text { section } \\
\text { letter }\end{array}$ & $\begin{array}{c}\text { Station }^{2} \\
\text { (feet) }\end{array}$ & $\begin{array}{l}\text { Width } \\
\text { (feet) }\end{array}$ & $\begin{array}{c}\text { Cross } \\
\text { sectional } \\
\text { area } \\
\text { (square feet) }\end{array}$ & $\begin{array}{c}\text { Mean } \\
\text { velocity } \\
\text { (feet per } \\
\text { second) }\end{array}$ & $\begin{array}{l}\text { Modeled stream with } \\
\text { BW (if any) from } \\
\text { receiving stream } \\
\text { (feet, NAVD 88) }\end{array}$ & $\begin{array}{l}\text { Modeled stream } \\
\text { without BW from } \\
\text { receiving stream } \\
\text { (feet, NAVD 88) }\end{array}$ & $\begin{array}{l}\text { Modeled stream with } \\
\text { floodway without BW } \\
\text { from receiving stream } \\
\text { (feet, NAVD 88) }\end{array}$ & $\begin{array}{c}\text { Increase in water surface } \\
\text { (without BW) } \\
\text { due to } \\
\text { floodway (feet) }\end{array}$ \\
\hline $\mathrm{AC}$ & 34,348 & 154 & 1,947 & 4.6 & 953.6 & 953.6 & 954.4 & 0.8 \\
\hline $\mathrm{AD}$ & 34,776 & 450 & 3,238 & 2.8 & 954.1 & 954.1 & 954.8 & 0.7 \\
\hline $\mathrm{AE}$ & 35,181 & 700 & 5,134 & 1.7 & 954.2 & 954.2 & 955.0 & 0.8 \\
\hline $\mathrm{AF}$ & 39,294 & 1,600 & 8,940 & 1.0 & 954.4 & 954.4 & 955.3 & 0.9 \\
\hline $\mathrm{AG}$ & 41,321 & 151 & 1,905 & 4.7 & 954.7 & 954.7 & 955.6 & 0.9 \\
\hline $\mathrm{AH}$ & 41,377 & 151 & 1,916 & 4.7 & 954.8 & 954.8 & 955.7 & 0.9 \\
\hline AI & 41,438 & 230 & 2,429 & 3.7 & 954.9 & 954.9 & 955.8 & 0.9 \\
\hline $\mathrm{AJ}$ & 42,226 & 400 & 3,126 & 2.2 & 955.4 & 955.4 & 956.2 & 0.8 \\
\hline $\mathrm{AK}$ & 46,739 & 662 & 2,684 & 2.1 & 955.8 & 955.8 & 956.7 & 0.9 \\
\hline $\mathrm{AL}$ & 46,788 & 506 & 2,283 & 2.1 & 955.8 & 955.8 & 956.7 & 0.9 \\
\hline $\mathrm{AM}$ & 47,369 & 1,200 & 6,193 & 0.7 & 955.9 & 955.9 & 956.9 & 1.0 \\
\hline $\mathrm{AN}$ & 53,306 & 1,700 & 6,779 & 0.7 & 956.2 & 956.2 & 957.1 & 0.9 \\
\hline $\mathrm{AO}$ & 53,329 & 1,700 & 6,722 & 0.7 & 956.2 & 956.2 & 957.1 & 0.9 \\
\hline AP & 54,107 & 1,409 & 3,996 & 1.0 & 956.3 & 956.3 & 957.2 & 0.9 \\
\hline AQ & 55,991 & 1,451 & 3,428 & 1.1 & 956.9 & 956.9 & 957.5 & 0.6 \\
\hline $\mathrm{AR}$ & 56,023 & 1,500 & 3,973 & 1.0 & 957.2 & 957.2 & 958.1 & 0.9 \\
\hline AS & 58,759 & 1,730 & 3,275 & 1.0 & 957.7 & 957.7 & 958.4 & 0.7 \\
\hline AT & 58,790 & 1,733 & 3,429 & 1.0 & 957.8 & 957.8 & 958.7 & 0.9 \\
\hline AU & 61,585 & 980 & 1,950 & 1.8 & 958.5 & 958.5 & 959.4 & 0.9 \\
\hline AV & 61,621 & 1,058 & 1,919 & 2.0 & 958.9 & 958.9 & 959.5 & 0.6 \\
\hline AW & 62,957 & 305 & 709 & 4.7 & 960.0 & 960.0 & 960.6 & 0.6 \\
\hline $\mathrm{AX}$ & 63,398 & 300 & 764 & 4.4 & 960.7 & 960.7 & 961.1 & 0.4 \\
\hline AY & 63,869 & 169 & 496 & 6.7 & 961.2 & 961.2 & 961.7 & 0.5 \\
\hline $\mathrm{AZ}$ & 64,302 & 175 & 585 & 5.7 & 962.2 & 962.2 & 962.5 & 0.3 \\
\hline BA & 64,644 & 125 & 573 & 5.8 & 962.5 & 962.5 & 963.0 & 0.5 \\
\hline BB & 64,690 & 101 & 653 & 5.1 & 962.9 & 962.9 & 963.3 & 0.4 \\
\hline $\mathrm{BC}$ & 64,721 & 103 & 665 & 5.0 & 962.9 & 962.9 & 963.4 & 0.5 \\
\hline $\mathrm{BD}$ & 64,937 & 190 & 792 & 4.2 & 963.5 & 963.5 & 963.7 & 0.2 \\
\hline
\end{tabular}


Table 1.1. Floodway data table for Chippewa Creek, Wayne and Medina Counties, Ohio._- Continued

[\%, percent; AEP, annual exceedance probability; BW, backwater; NAVD 88, North American Vertical Datum of 1988]

\begin{tabular}{|c|c|c|c|c|c|c|c|c|}
\hline \multicolumn{2}{|c|}{ Location } & \multicolumn{3}{|c|}{ Floodway $^{1}$} & \multicolumn{4}{|c|}{$1 \%$ AEP water-surface elevation } \\
\hline $\begin{array}{l}\text { Cross } \\
\text { section } \\
\text { letter }\end{array}$ & $\begin{array}{c}\text { Station }^{2} \\
\text { (feet) }\end{array}$ & $\begin{array}{l}\text { Width } \\
\text { (feet) }\end{array}$ & $\begin{array}{c}\text { Cross } \\
\text { sectional } \\
\text { area } \\
\text { (square feet) }\end{array}$ & $\begin{array}{l}\text { Mean } \\
\text { velocity } \\
\text { (feet per } \\
\text { second) }\end{array}$ & $\begin{array}{l}\text { Modeled stream with } \\
\text { BW (if any) from } \\
\text { receiving stream } \\
\text { (feet, NAVD 88) }\end{array}$ & $\begin{array}{l}\text { Modeled stream } \\
\text { without BW from } \\
\text { receiving stream } \\
\text { (feet, NAVD 88) }\end{array}$ & $\begin{array}{l}\text { Modeled stream with } \\
\text { floodway without BW } \\
\text { from receiving stream } \\
\text { (feet, NAVD 88) }\end{array}$ & $\begin{array}{c}\text { Increase in water surface } \\
\text { (without BW) } \\
\text { due to } \\
\text { floodway (feet) }\end{array}$ \\
\hline $\mathrm{BE}$ & 66,365 & 690 & 1,680 & 2.0 & 964.1 & 964.1 & 964.7 & 0.6 \\
\hline $\mathrm{BF}$ & 66,404 & 690 & 1,887 & 1.8 & 964.1 & 964.1 & 965.0 & 0.9 \\
\hline BG & 66,782 & 550 & 1,402 & 2.4 & 964.1 & 964.1 & 965.1 & 1.0 \\
\hline $\mathrm{BH}$ & 67,142 & 500 & 1,260 & 2.7 & 964.4 & 964.4 & 965.2 & 0.8 \\
\hline $\mathrm{BI}$ & 69,018 & 410 & 1,015 & 1.8 & 965.1 & 965.1 & 965.9 & 0.8 \\
\hline $\mathrm{BJ}$ & 69,045 & 410 & 1,038 & 1.8 & 965.1 & 965.1 & 966.0 & 0.9 \\
\hline $\mathrm{BK}$ & 69,879 & 250 & 764 & 2.4 & 965.3 & 965.3 & 966.2 & 0.9 \\
\hline $\mathrm{BL}$ & 70,700 & 110 & 521 & 3.5 & 965.7 & 965.7 & 966.6 & 0.9 \\
\hline $\mathrm{BM}$ & 71,195 & 81 & 564 & 3.3 & 966.2 & 966.2 & 967.1 & 0.9 \\
\hline $\mathrm{BN}$ & 71,253 & 93 & 600 & 3.1 & 966.4 & 966.4 & 967.2 & 0.8 \\
\hline $\mathrm{BO}$ & 72,487 & 150 & 539 & 3.4 & 967.2 & 967.2 & 967.8 & 0.6 \\
\hline $\mathrm{BP}$ & 73,271 & 125 & 631 & 2.9 & 967.6 & 967.6 & 968.6 & 1.0 \\
\hline BQ & 73,953 & 100 & 565 & 3.3 & 968.0 & 968.0 & 968.9 & 0.9 \\
\hline BR & 74,557 & 70 & 411 & 4.5 & 968.6 & 968.6 & 969.3 & 0.7 \\
\hline BS & 74,747 & 70 & 498 & 3.7 & 969.0 & 969.0 & 969.5 & 0.5 \\
\hline BT & 75,501 & 56 & 364 & 5.1 & 969.3 & 969.3 & 969.8 & 0.5 \\
\hline $\mathrm{BU}$ & 75,547 & 56 & 368 & 5.0 & 969.4 & 969.4 & 969.9 & 0.5 \\
\hline BV & 75,599 & 60 & 364 & 5.0 & 969.6 & 969.6 & 970.0 & 0.4 \\
\hline BW & 76,468 & 75 & 426 & 4.3 & 970.8 & 970.8 & 971.1 & 0.3 \\
\hline $\mathrm{BX}$ & 78,529 & 85 & 456 & 3.8 & 972.1 & 972.1 & 972.8 & 0.7 \\
\hline BY & 78,890 & 80 & 430 & 4.0 & 972.6 & 972.6 & 973.0 & 0.4 \\
\hline $\mathrm{BZ}$ & 80,625 & 80 & 363 & 4.8 & 974.2 & 974.2 & 974.6 & 0.4 \\
\hline $\mathrm{CA}$ & 82,428 & 80 & 375 & 4.6 & 977.2 & 977.2 & 977.3 & 0.1 \\
\hline $\mathrm{CB}$ & 82,776 & 80 & 349 & 5.0 & 977.6 & 977.6 & 977.8 & 0.2 \\
\hline $\mathrm{CC}$ & 83,564 & 47 & 343 & 5.1 & 979.2 & 979.2 & 979.3 & 0.1 \\
\hline $\mathrm{CD}$ & 83,594 & 47 & 351 & 5.0 & 979.3 & 979.3 & 979.5 & 0.2 \\
\hline $\mathrm{CE}$ & 83,937 & 67 & 393 & 4.4 & 979.9 & 979.9 & 980.0 & 0.1 \\
\hline $\mathrm{CF}$ & 84,627 & 65 & 407 & 4.3 & 980.7 & 980.7 & 980.8 & 0.1 \\
\hline
\end{tabular}


Table 1.1. Floodway data table for Chippewa Creek, Wayne and Medina Counties, Ohio.-Continued

[\%, percent; AEP, annual exceedance probability; BW, backwater; NAVD 88, North American Vertical Datum of 1988]

\begin{tabular}{|c|c|c|c|c|c|c|c|c|}
\hline \multicolumn{2}{|c|}{ Location } & \multicolumn{3}{|c|}{ Floodway $^{1}$} & \multicolumn{4}{|c|}{$1 \%$ AEP water-surface elevation } \\
\hline $\begin{array}{l}\text { Cross } \\
\text { section } \\
\text { letter }\end{array}$ & $\begin{array}{c}\text { Station }^{2} \\
\text { (feet) }\end{array}$ & $\begin{array}{l}\text { Width } \\
\text { (feet) }\end{array}$ & $\begin{array}{c}\text { Cross } \\
\text { sectional } \\
\text { area } \\
\text { (square feet) }\end{array}$ & $\begin{array}{c}\text { Mean } \\
\text { velocity } \\
\text { (feet per } \\
\text { second) }\end{array}$ & $\begin{array}{l}\text { Modeled stream with } \\
\text { BW (if any) from } \\
\text { receiving stream } \\
\text { (feet, NAVD 88) }\end{array}$ & $\begin{array}{l}\text { Modeled stream } \\
\text { without BW from } \\
\text { receiving stream } \\
\text { (feet, NAVD 88) }\end{array}$ & $\begin{array}{l}\text { Modeled stream with } \\
\text { floodway without BW } \\
\text { from receiving stream } \\
\text { (feet, NAVD 88) }\end{array}$ & $\begin{array}{c}\text { Increase in water surface } \\
\text { (without BW) } \\
\text { due to } \\
\text { floodway (feet) }\end{array}$ \\
\hline $\mathrm{CG}$ & 85,236 & 57 & 371 & 4.7 & 981.2 & 981.2 & 981.5 & 0.3 \\
\hline $\mathrm{CH}$ & 85,614 & 57 & 392 & 4.4 & 981.8 & 981.8 & 982.0 & 0.2 \\
\hline $\mathrm{CI}$ & 85,628 & 57 & 395 & 4.4 & 982.0 & 982.0 & 982.1 & 0.1 \\
\hline $\mathrm{CJ}$ & 85,993 & 50 & 263 & 6.6 & 982.3 & 982.3 & 982.4 & 0.1 \\
\hline CK & 86,051 & 51 & 248 & 7.0 & 982.4 & 982.4 & 982.5 & 0.1 \\
\hline $\mathrm{CL}$ & 86,116 & 45 & 319 & 5.4 & 982.9 & 982.9 & 983.0 & 0.1 \\
\hline $\mathrm{CM}$ & 86,475 & 55 & 367 & 4.1 & 983.6 & 983.6 & 983.7 & 0.1 \\
\hline $\mathrm{CN}$ & 86,852 & 80 & 578 & 2.6 & 983.9 & 983.9 & 984.1 & 0.2 \\
\hline $\mathrm{CO}$ & 88,898 & 100 & 684 & 2.2 & 984.3 & 984.3 & 984.5 & 0.2 \\
\hline $\mathrm{CP}$ & 88,914 & 100 & 685 & 2.2 & 984.3 & 984.3 & 984.5 & 0.2 \\
\hline CQ & 91,024 & 70 & 563 & 2.6 & 984.5 & 984.5 & 984.9 & 0.4 \\
\hline $\mathrm{CR}$ & 91,063 & 70 & 572 & 2.6 & 984.5 & 984.5 & 984.9 & 0.4 \\
\hline $\mathrm{CS}$ & 91,461 & 120 & 859 & 1.7 & 984.7 & 984.7 & 985.1 & 0.4 \\
\hline $\mathrm{CT}$ & 92,060 & 150 & 924 & 1.6 & 984.8 & 984.8 & 985.2 & 0.4 \\
\hline $\mathrm{CU}$ & 95,932 & 62 & 358 & 4.0 & 984.9 & 984.9 & 985.7 & 0.8 \\
\hline $\mathrm{CV}$ & 96,088 & 50 & 308 & 4.7 & 985.2 & 985.2 & 985.9 & 0.7 \\
\hline $\mathrm{CW}$ & 96,297 & 75 & 439 & 3.3 & 985.7 & 985.7 & 986.2 & 0.5 \\
\hline $\mathrm{CX}$ & 96,450 & 76 & 412 & 3.5 & 985.7 & 985.7 & 986.3 & 0.6 \\
\hline $\mathrm{CY}$ & 96,611 & 76 & 420 & 3.4 & 985.9 & 985.9 & 986.4 & 0.5 \\
\hline $\mathrm{CZ}$ & 96,891 & 135 & 557 & 2.6 & 986.3 & 986.3 & 986.6 & 0.3 \\
\hline DA & 99,695 & 130 & 463 & 2.7 & 986.7 & 986.7 & 987.7 & 1.0 \\
\hline DB & 100,505 & 42 & 258 & 4.8 & 987.8 & 987.8 & 988.5 & 0.7 \\
\hline DC & 101,334 & 39 & 248 & 5.0 & 988.7 & 988.7 & 989.3 & 0.6 \\
\hline DD & 101,821 & 26 & 189 & 6.5 & 989.1 & 989.1 & 989.6 & 0.5 \\
\hline $\mathrm{DE}$ & 101,850 & 33 & 219 & 5.6 & 989.3 & 989.3 & 989.8 & 0.5 \\
\hline DF & 101,868 & 45 & 257 & 4.8 & 989.5 & 989.5 & 990.0 & 0.5 \\
\hline DG & 102,400 & 48 & 288 & 4.3 & 990.4 & 990.4 & 990.9 & 0.5 \\
\hline $\mathrm{DH}$ & 103,298 & 63 & 379 & 3.2 & 991.5 & 991.5 & 991.9 & 0.4 \\
\hline
\end{tabular}


Table 1.1. Floodway data table for Chippewa Creek, Wayne and Medina Counties, Ohio.-Continued

[\%, percent; AEP, annual exceedance probability; BW, backwater; NAVD 88, North American Vertical Datum of 1988]

\begin{tabular}{|c|c|c|c|c|c|c|c|c|}
\hline \multicolumn{2}{|c|}{ Location } & \multicolumn{3}{|c|}{ Floodway ${ }^{1}$} & \multicolumn{4}{|c|}{$1 \%$ AEP water-surface elevation } \\
\hline $\begin{array}{l}\text { Cross } \\
\text { section } \\
\text { letter }\end{array}$ & $\begin{array}{c}\text { Station }^{2} \\
\text { (feet) }\end{array}$ & $\begin{array}{l}\text { Width } \\
\text { (feet) }\end{array}$ & $\begin{array}{c}\text { Cross } \\
\text { sectional } \\
\text { area } \\
\text { (square feet) }\end{array}$ & $\begin{array}{l}\text { Mean } \\
\text { velocity } \\
\text { (feet per } \\
\text { second) }\end{array}$ & $\begin{array}{l}\text { Modeled stream with } \\
\text { BW (if any) from } \\
\text { receiving stream } \\
\text { (feet, NAVD 88) }\end{array}$ & $\begin{array}{l}\text { Modeled stream } \\
\text { without BW from } \\
\text { receiving stream } \\
\text { (feet, NAVD 88) }\end{array}$ & $\begin{array}{l}\text { Modeled stream with } \\
\text { floodway without BW } \\
\text { from receiving stream } \\
\text { (feet, NAVD 88) }\end{array}$ & $\begin{array}{c}\text { Increase in water surface } \\
\text { (without BW) } \\
\text { due to } \\
\text { floodway (feet) }\end{array}$ \\
\hline DI & 104,495 & 71 & 468 & 2.4 & 992.0 & 992.0 & 992.7 & 0.7 \\
\hline DJ & 104,538 & 71 & 474 & 2.3 & 992.1 & 992.1 & 992.7 & 0.6 \\
\hline DK & 104,550 & 71 & 606 & 1.8 & 992.1 & 992.1 & 992.8 & 0.7 \\
\hline DL & 114,578 & 800 & 3,831 & 0.3 & 992.3 & 992.3 & 993.0 & 0.7 \\
\hline $\mathrm{DM}$ & 114,610 & 800 & 3,608 & 0.3 & 992.3 & 992.3 & 993.0 & 0.7 \\
\hline $\mathrm{DN}$ & 120,793 & 75 & 384 & 2.9 & 992.4 & 992.4 & 993.2 & 0.8 \\
\hline $\mathrm{DO}$ & 121,461 & 42 & 230 & 4.8 & 992.7 & 992.7 & 993.4 & 0.7 \\
\hline DP & 121,832 & 39 & 200 & 5.5 & 993.1 & 993.1 & 993.7 & 0.6 \\
\hline DQ & 122,490 & 45 & 220 & 5.0 & 994.4 & 994.4 & 994.6 & 0.2 \\
\hline $\mathrm{DR}$ & 122,987 & 43 & 199 & 5.6 & 995.1 & 995.1 & 995.3 & 0.2 \\
\hline DS & 123,049 & 47 & 203 & 5.5 & 995.3 & 995.3 & 995.4 & 0.1 \\
\hline DT & 123,099 & 50 & 222 & 5.0 & 995.7 & 995.7 & 995.8 & 0.1 \\
\hline $\mathrm{DU}$ & 123,373 & 39 & 234 & 4.7 & 996.3 & 996.3 & 996.3 & 0.0 \\
\hline DV & 124,337 & 35 & 203 & 5.5 & 997.7 & 997.7 & 998.3 & 0.6 \\
\hline
\end{tabular}

${ }^{1}$ A floodway is defined as the channel of a river or other watercourse and the adjacent land areas that must be reserved to discharge the 1-percent annual exceedance probability flood without increasing the water-surface elevation more than a designated height (Federal Emergency Management Agency, 2020).

${ }^{2}$ Station is the distance in feet upstream from stream mouth. 
Table 1.2. Floodway data table for Little Chippewa Creek, Wayne County, Ohio.

[\%, percent; AEP, annual exceedance probability; BW, backwater; NAVD 88, North American Vertical Datum of 1988]

\begin{tabular}{|c|c|c|c|c|c|c|c|c|}
\hline \multicolumn{2}{|c|}{ Location } & \multicolumn{3}{|c|}{ Floodway $^{1}$} & \multicolumn{4}{|c|}{$1 \%$ AEP water-surface elevation } \\
\hline $\begin{array}{l}\text { Cross } \\
\text { section } \\
\text { letter }\end{array}$ & $\begin{array}{c}\text { Station }^{2} \\
\text { (feet) }\end{array}$ & $\begin{array}{l}\text { Width } \\
\text { (feet) }\end{array}$ & $\begin{array}{c}\text { Cross } \\
\text { sectional } \\
\text { area } \\
\text { (square feet) }\end{array}$ & $\begin{array}{c}\text { Mean } \\
\text { velocity } \\
\text { (feet per } \\
\text { second) }\end{array}$ & $\begin{array}{l}\text { Modeled stream with } \\
\text { BW (if any) from } \\
\text { receiving stream } \\
\text { (feet, NAVD 88) }\end{array}$ & $\begin{array}{l}\text { Modeled stream } \\
\text { without BW from } \\
\text { receiving stream } \\
\text { (feet, NAVD 88) }\end{array}$ & $\begin{array}{l}\text { Modeled stream with } \\
\text { floodway without BW } \\
\text { from receiving stream } \\
\text { (feet, NAVD 88) }\end{array}$ & $\begin{array}{c}\text { Increase in water surface } \\
\text { (without BW) } \\
\text { due to } \\
\text { floodway (feet) }\end{array}$ \\
\hline A & 88 & 57 & 434 & 6.4 & ${ }^{3} 956.8$ & 951.1 & 951.4 & 0.3 \\
\hline B & 824 & 70 & 374 & 7.4 & ${ }^{3} 956.8$ & 952.7 & 952.9 & 0.2 \\
\hline $\mathrm{C}$ & 886 & 105 & 524 & 5.3 & ${ }^{3} 956.8$ & 953.4 & 953.6 & 0.2 \\
\hline D & 963 & 105 & 550 & 5.1 & ${ }^{3} 956.8$ & 953.7 & 953.8 & 0.1 \\
\hline $\mathrm{E}$ & 1,018 & 325 & 969 & 2.9 & ${ }^{3} 956.8$ & 954.1 & 954.1 & 0.0 \\
\hline $\mathrm{F}$ & 1,515 & 375 & 1,075 & 2.6 & ${ }^{3} 956.8$ & 954.5 & 954.5 & 0.0 \\
\hline G & 2,799 & 486 & 2,064 & 1.3 & ${ }^{3} 956.8$ & 954.8 & 955.3 & 0.5 \\
\hline $\mathrm{H}$ & 3,597 & 600 & 2,057 & 1.4 & ${ }^{3} 956.8$ & 954.8 & 955.5 & 0.7 \\
\hline I & 3,631 & 600 & 1,932 & 1.4 & ${ }^{3} 956.8$ & 954.8 & 955.5 & 0.7 \\
\hline $\mathrm{J}$ & 5,588 & 750 & 3,153 & 0.8 & ${ }^{3} 956.8$ & 954.9 & 955.8 & 0.9 \\
\hline K & 5,990 & 750 & 2,967 & 0.8 & ${ }^{3} 956.8$ & 955.0 & 955.9 & 0.9 \\
\hline $\mathrm{L}$ & 7,357 & 750 & 2,784 & 0.9 & ${ }^{3} 956.8$ & 955.0 & 956.0 & 1.0 \\
\hline $\mathrm{M}$ & 7,637 & 800 & 3,264 & 0.7 & ${ }^{3} 956.8$ & 955.1 & 956.1 & 1.0 \\
\hline $\mathrm{N}$ & 8,386 & 850 & 2,260 & 1.1 & ${ }^{3} 956.8$ & 955.1 & 956.1 & 1.0 \\
\hline
\end{tabular}


Table 1.2. Floodway data table for Little Chippewa Creek, Wayne County, Ohio.-Continued

[\%, percent; AEP, annual exceedance probability; BW, backwater; NAVD 88, North American Vertical Datum of 1988]

\begin{tabular}{|c|c|c|c|c|c|c|c|c|}
\hline \multicolumn{2}{|c|}{ Location } & \multicolumn{3}{|c|}{ Floodway $^{1}$} & \multicolumn{4}{|c|}{$1 \%$ AEP water-surface elevation } \\
\hline $\begin{array}{l}\text { Cross } \\
\text { section } \\
\text { letter }\end{array}$ & $\begin{array}{c}\text { Station }^{2} \\
\text { (feet) }\end{array}$ & $\begin{array}{l}\text { Width } \\
\text { (feet) }\end{array}$ & $\begin{array}{c}\text { Cross } \\
\text { sectional } \\
\text { area } \\
\text { (square feet) }\end{array}$ & $\begin{array}{c}\text { Mean } \\
\text { velocity } \\
\text { (feet per } \\
\text { second) }\end{array}$ & $\begin{array}{l}\text { Modeled stream with } \\
\text { BW (if any) from } \\
\text { receiving stream } \\
\text { (feet, NAVD 88) }\end{array}$ & $\begin{array}{l}\text { Modeled stream } \\
\text { without BW from } \\
\text { receiving stream } \\
\text { (feet, NAVD 88) }\end{array}$ & $\begin{array}{l}\text { Modeled stream with } \\
\text { floodway without BW } \\
\text { from receiving stream } \\
\text { (feet, NAVD 88) }\end{array}$ & $\begin{array}{c}\text { Increase in water surface } \\
\text { (without BW) } \\
\text { due to } \\
\text { floodway (feet) }\end{array}$ \\
\hline $\mathrm{O}$ & 9,304 & 1,050 & 2,560 & 1.5 & ${ }^{3} 956.8$ & 955.4 & 956.3 & 0.9 \\
\hline$P$ & 9,579 & 1,190 & 2,805 & 1.6 & ${ }^{3} 956.8$ & 955.4 & 956.4 & 1.0 \\
\hline Q & 10,511 & 1,493 & 3,756 & 1.9 & ${ }^{3} 956.8$ & 955.9 & 956.8 & 0.9 \\
\hline $\mathrm{R}$ & 11,540 & 1,682 & 3,959 & 3.0 & ${ }^{3} 956.8$ & 956.7 & 957.6 & 0.9 \\
\hline $\mathrm{S}$ & 11,634 & 1,987 & 5,761 & 2.8 & 957.3 & 957.3 & 957.8 & 0.5 \\
\hline $\mathrm{T}$ & 12,065 & 859 & 1,026 & 4.6 & 957.6 & 957.6 & 958.3 & 0.7 \\
\hline $\mathrm{U}$ & 12,705 & 400 & 1,006 & 2.8 & 958.7 & 958.7 & 959.4 & 0.7 \\
\hline V & 13,024 & 250 & 784 & 3.0 & 959.0 & 959.0 & 959.7 & 0.7 \\
\hline W & 13,573 & 175 & 713 & 3.3 & 959.8 & 959.8 & 960.2 & 0.4 \\
\hline $\mathrm{X}$ & 15,507 & 196 & 752 & 3.2 & 961.0 & 961.0 & 961.7 & 0.7 \\
\hline Y & 15,805 & 222 & 688 & 3.5 & 961.1 & 961.1 & 961.9 & 0.8 \\
\hline Z & 17,040 & 270 & 812 & 2.9 & 961.8 & 961.8 & 962.7 & 0.9 \\
\hline $\mathrm{AA}$ & 17,552 & 250 & 651 & 3.7 & 962.1 & 962.1 & 963.0 & 0.9 \\
\hline $\mathrm{AB}$ & 18,186 & 250 & 717 & 3.2 & 962.8 & 962.8 & 963.6 & 0.8 \\
\hline
\end{tabular}

${ }^{1}$ A floodway is defined as the channel of a river or other watercourse and the adjacent land areas that must be reserved to discharge the 1-percent annual exceedance probability flood without increasing the water-surface elevation more than a designated height (Federal Emergency Management Agency, 2020).

${ }^{2}$ Station is the distance in feet upstream from stream mouth.

${ }^{3}$ Backwater elevation from river station 55,585 of the Chippewa Creek model. 
Table 1.3. Floodway data table for Styx River, Wayne and Medina Counties, Ohio.

[\%, percent; AEP, annual exceedance probability; BW, backwater; NAVD 88, North American Vertical Datum of 1988]

\begin{tabular}{|c|c|c|c|c|c|c|c|c|}
\hline \multicolumn{2}{|c|}{ Location } & \multicolumn{3}{|c|}{ Floodway $^{1}$} & \multicolumn{4}{|c|}{$1 \%$ AEP water-surface elevation } \\
\hline $\begin{array}{l}\text { Cross } \\
\text { section } \\
\text { letter }\end{array}$ & $\begin{array}{c}\text { Station }^{2} \\
\text { (feet) }\end{array}$ & $\begin{array}{c}\text { Width, } \\
\text { (feet) }\end{array}$ & $\begin{array}{c}\text { Cross } \\
\text { sectional } \\
\text { area } \\
\text { (square feet) }\end{array}$ & $\begin{array}{l}\text { Mean } \\
\text { velocity } \\
\text { (feet per } \\
\text { second) }\end{array}$ & $\begin{array}{l}\text { Modeled stream with BW } \\
\text { (if any) from } \\
\text { receiving stream } \\
\text { (feet, NAVD 88) }\end{array}$ & $\begin{array}{l}\text { Modeled stream } \\
\text { without BW from } \\
\text { receiving stream } \\
\text { (feet, NAVD 88) }\end{array}$ & $\begin{array}{l}\text { Modeled stream with } \\
\text { floodway without BW } \\
\text { from receiving stream } \\
\text { (feet, NAVD 88) }\end{array}$ & $\begin{array}{c}\text { Increase in water surface } \\
\text { (without BW) } \\
\text { due to } \\
\text { floodway (feet) }\end{array}$ \\
\hline $\mathrm{A}$ & 189 & 70 & 523 & 5.7 & $955.6^{3}$ & 947.9 & 947.9 & 0.0 \\
\hline $\mathrm{B}$ & 1,172 & 85 & 615 & 4.8 & $955.6^{3}$ & 949.3 & 949.3 & 0.0 \\
\hline $\mathrm{C}$ & 2,469 & 88 & 666 & 4.5 & $955.6^{3}$ & 950.8 & 950.8 & 0.0 \\
\hline $\mathrm{D}$ & 3,818 & 52 & 484 & 6.1 & $955.6^{3}$ & 951.9 & 952.0 & 0.1 \\
\hline E & 3,866 & 52 & 488 & 6.1 & $955.6^{3}$ & 952.0 & 952.1 & 0.1 \\
\hline $\mathrm{F}$ & 3,924 & 55 & 407 & 7.3 & $955.6^{3}$ & 952.0 & 952.1 & 0.1 \\
\hline G & 3,957 & 55 & 413 & 7.2 & $955.6^{3}$ & 952.1 & 952.2 & 0.1 \\
\hline $\mathrm{H}$ & 3,990 & 61 & 515 & 5.8 & $955.6^{3}$ & 952.5 & 952.6 & 0.1 \\
\hline I & 4,016 & 60 & 517 & 5.7 & $955.6^{3}$ & 952.6 & 952.7 & 0.1 \\
\hline $\mathrm{J}$ & 4,078 & 59 & 524 & 5.7 & $955.6^{3}$ & 952.7 & 952.8 & 0.1 \\
\hline $\mathrm{K}$ & 4,109 & 59 & 527 & 5.6 & $955.6^{3}$ & 952.7 & 952.8 & 0.1 \\
\hline $\mathrm{L}$ & 4,291 & 63 & 537 & 5.5 & $955.6^{3}$ & 953.0 & 953.1 & 0.1 \\
\hline M & 4,344 & 66 & 553 & 5.4 & $955.6^{3}$ & 953.2 & 953.2 & 0.0 \\
\hline $\mathrm{N}$ & 4,710 & 73 & 642 & 4.6 & $955.6^{3}$ & 953.7 & 953.7 & 0.0 \\
\hline $\mathrm{O}$ & 5,433 & 69 & 559 & 5.3 & $955.6^{3}$ & 954.3 & 954.3 & 0.0 \\
\hline $\mathrm{P}$ & 5,471 & 70 & 569 & 5.2 & $955.6^{3}$ & 954.4 & 954.5 & 0.1 \\
\hline Q & 6,358 & 91 & 618 & 4.8 & $955.6^{3}$ & 955.2 & 955.2 & 0.0 \\
\hline $\mathrm{R}$ & 6,387 & 86 & 599 & 5.0 & $955.6^{3}$ & 955.2 & 955.3 & 0.1 \\
\hline $\mathrm{S}$ & 6,466 & 94 & 713 & 4.2 & $955.6^{3}$ & 955.5 & 955.5 & 0.0 \\
\hline $\mathrm{T}$ & 6,514 & 94 & 722 & 4.1 & $955.6^{3}$ & 955.6 & 955.6 & 0.0 \\
\hline $\mathrm{U}$ & 6,911 & 250 & 1,264 & 2.3 & 955.9 & 955.9 & 955.9 & 0.0 \\
\hline $\mathrm{V}$ & 8,893 & 250 & 949 & 3.0 & 956.3 & 956.3 & 956.6 & 0.3 \\
\hline W & 8,959 & 250 & 1,140 & 2.5 & 956.9 & 956.9 & 957.3 & 0.4 \\
\hline $\mathrm{X}$ & 9,609 & 400 & 1,641 & 1.7 & 957.2 & 957.2 & 957.6 & 0.4 \\
\hline $\mathrm{Y}$ & 10,586 & 450 & 1,680 & 1.7 & 957.3 & 957.3 & 957.9 & 0.6 \\
\hline $\mathrm{Z}$ & 11,943 & 450 & 1,255 & 2.2 & 957.8 & 957.8 & 958.6 & 0.8 \\
\hline AA & 12,016 & 450 & 1,548 & 1.8 & 958.8 & 958.8 & 959.6 & 0.8 \\
\hline $\mathrm{AB}$ & 14,828 & 650 & 1,532 & 1.8 & 959.3 & 959.3 & 960.2 & 0.9 \\
\hline
\end{tabular}


Table 1.3. Floodway data table for Styx River, Wayne and Medina Counties, Ohio.-Continued

[\%, percent; AEP, annual exceedance probability; BW, backwater; NAVD 88, North American Vertical Datum of 1988]

\begin{tabular}{|c|c|c|c|c|c|c|c|c|}
\hline \multicolumn{2}{|c|}{ Location } & \multicolumn{3}{|c|}{ Floodway $^{1}$} & \multicolumn{4}{|c|}{$1 \%$ AEP water-surface elevation } \\
\hline $\begin{array}{l}\text { Cross } \\
\text { section } \\
\text { letter }\end{array}$ & $\begin{array}{c}\text { Station }^{2} \\
\text { (feet) }\end{array}$ & $\begin{array}{c}\text { Width, } \\
\text { (feet) }\end{array}$ & $\begin{array}{c}\text { Cross } \\
\text { sectional } \\
\text { area } \\
\text { (square feet) }\end{array}$ & $\begin{array}{l}\text { Mean } \\
\text { velocity } \\
\text { (feet per } \\
\text { second) }\end{array}$ & $\begin{array}{l}\text { Modeled stream with } \\
\text { BW (if any) from } \\
\text { receiving stream } \\
\text { (feet, NAVD 88) }\end{array}$ & $\begin{array}{l}\text { Modeled stream } \\
\text { without BW from } \\
\text { receiving stream } \\
\text { (feet, NAVD 88) }\end{array}$ & $\begin{array}{l}\text { Modeled stream with } \\
\text { floodway without BW } \\
\text { from receiving stream } \\
\text { (feet, NAVD 88) }\end{array}$ & $\begin{array}{c}\text { Increase in water surface } \\
\text { (without BW) } \\
\text { due to } \\
\text { floodway (feet) }\end{array}$ \\
\hline $\mathrm{AC}$ & 14,874 & 592 & 1,804 & 1.6 & 959.8 & 959.8 & 960.8 & 1.0 \\
\hline $\mathrm{AD}$ & 15,805 & 400 & 1,309 & 2.1 & 960.1 & 960.1 & 961.1 & 1.0 \\
\hline $\mathrm{AE}$ & 16,358 & 250 & 688 & 4.1 & 960.4 & 960.4 & 961.3 & 0.9 \\
\hline $\mathrm{AF}$ & 16,682 & 165 & 584 & 4.8 & 961.0 & 961.0 & 961.7 & 0.7 \\
\hline $\mathrm{AG}$ & 17,358 & 100 & 489 & 5.7 & 961.7 & 961.7 & 962.4 & 0.7 \\
\hline $\mathrm{AH}$ & 17,529 & 150 & 583 & 4.8 & 962.2 & 962.2 & 962.8 & 0.6 \\
\hline AI & 17,624 & 150 & 619 & 4.5 & 962.7 & 962.7 & 962.9 & 0.2 \\
\hline AJ & 17,786 & 250 & 1,439 & 2.0 & 963.1 & 963.1 & 963.5 & 0.4 \\
\hline AK & 17,801 & 250 & 1,312 & 2.1 & 963.2 & 963.2 & 963.5 & 0.3 \\
\hline $\mathrm{AL}$ & 19,052 & 400 & 1,723 & 1.1 & 963.4 & 963.4 & 964.1 & 0.7 \\
\hline $\mathrm{AM}$ & 20,521 & 600 & 1,767 & 1.1 & 963.6 & 963.6 & 964.2 & 0.6 \\
\hline AN & 20,573 & 600 & 2,472 & 0.8 & 963.6 & 963.6 & 964.3 & 0.7 \\
\hline $\mathrm{AO}$ & 21,779 & 600 & 1,636 & 1.1 & 963.7 & 963.7 & 964.5 & 0.8 \\
\hline AP & 21,805 & 600 & 1,617 & 1.1 & 963.7 & 963.7 & 964.5 & 0.8 \\
\hline AQ & 22,235 & 550 & 1,534 & 1.1 & 963.8 & 963.8 & 964.5 & 0.7 \\
\hline AR & 22,805 & 450 & 1,340 & 1.3 & 963.9 & 963.9 & 964.7 & 0.8 \\
\hline AS & 23,466 & 400 & 818 & 2.2 & 964.1 & 964.1 & 965.0 & 0.9 \\
\hline AT & 24,408 & 254 & 507 & 3.5 & 965.0 & 965.0 & 965.6 & 0.6 \\
\hline $\mathrm{AU}$ & 25,507 & 300 & 546 & 3.2 & 966.0 & 966.0 & 966.8 & 0.8 \\
\hline $\mathrm{AV}$ & 26,148 & 300 & 771 & 2.2 & 966.8 & 966.8 & 967.7 & 0.9 \\
\hline AW & 27,085 & 120 & 437 & 3.9 & 967.7 & 967.7 & 968.5 & 0.8 \\
\hline $\mathrm{AX}$ & 27,268 & 53 & 214 & 8.0 & 968.1 & 968.1 & 968.6 & 0.5 \\
\hline AY & 27,341 & 52 & 257 & 6.6 & 968.7 & 968.7 & 969.6 & 0.9 \\
\hline $\mathrm{AZ}$ & 27,585 & 65 & 298 & 5.7 & 969.9 & 969.9 & 970.5 & 0.6 \\
\hline BA & 27,996 & 200 & 533 & 3.2 & 970.7 & 970.7 & 971.5 & 0.8 \\
\hline BB & 29,243 & 200 & 463 & 3.7 & 971.8 & 971.8 & 972.6 & 0.8 \\
\hline $\mathrm{BC}$ & 29,710 & 100 & 383 & 4.4 & 972.4 & 972.4 & 973.2 & 0.8 \\
\hline $\mathrm{BD}$ & 29,839 & 61 & 321 & 5.3 & 972.7 & 972.7 & 973.4 & 0.7 \\
\hline $\mathrm{BE}$ & 29,960 & 65 & 354 & 4.8 & 973.5 & 973.5 & 973.9 & 0.4 \\
\hline
\end{tabular}


Table 1.3. Floodway data table for Styx River, Wayne and Medina Counties, Ohio.—Continued

[\%, percent; AEP, annual exceedance probability; BW, backwater; NAVD 88, North American Vertical Datum of 1988]

\begin{tabular}{|c|c|c|c|c|c|c|c|c|}
\hline \multicolumn{2}{|c|}{ Location } & \multicolumn{3}{|c|}{ Floodway $^{1}$} & \multicolumn{4}{|c|}{$1 \%$ AEP water-surface elevation } \\
\hline $\begin{array}{l}\text { Cross } \\
\text { section } \\
\text { letter }\end{array}$ & $\begin{array}{c}\text { Station }^{2} \\
\text { (feet) }\end{array}$ & $\begin{array}{c}\text { Width, } \\
\text { (feet) }\end{array}$ & $\begin{array}{c}\text { Cross } \\
\text { sectional } \\
\text { area } \\
\text { (square feet) }\end{array}$ & $\begin{array}{c}\text { Mean } \\
\text { velocity } \\
\text { (feet per } \\
\text { second) }\end{array}$ & $\begin{array}{l}\text { Modeled stream with } \\
\text { BW (if any) from } \\
\text { receiving stream } \\
\text { (feet, NAVD 88) }\end{array}$ & $\begin{array}{l}\text { Modeled stream } \\
\text { without BW from } \\
\text { receiving stream } \\
\text { (feet, NAVD 88) }\end{array}$ & $\begin{array}{l}\text { Modeled stream with } \\
\text { floodway without BW } \\
\text { from receiving stream } \\
\text { (feet, NAVD 88) }\end{array}$ & $\begin{array}{c}\text { Increase in water surface } \\
\text { (without BW) } \\
\text { due to } \\
\text { floodway (feet) }\end{array}$ \\
\hline $\mathrm{BF}$ & 30,076 & 100 & 464 & 3.7 & 973.9 & 973.9 & 974.2 & 0.3 \\
\hline BG & 31,166 & 200 & 798 & 2.0 & 974.9 & 974.9 & 975.4 & 0.5 \\
\hline $\mathrm{BH}$ & 32,671 & 400 & 1,170 & 1.3 & 975.7 & 975.7 & 976.6 & 0.9 \\
\hline BI & 32,693 & 400 & 1,043 & 1.5 & 975.7 & 975.7 & 976.6 & 0.9 \\
\hline $\mathrm{BJ}$ & 33,199 & 275 & 565 & 2.8 & 976.2 & 976.2 & 977.2 & 1.0 \\
\hline $\mathrm{BK}$ & 33,442 & 200 & 521 & 3.0 & 976.8 & 976.8 & 977.5 & 0.7 \\
\hline $\mathrm{BL}$ & 34,071 & 200 & 394 & 4.0 & 977.9 & 977.9 & 978.4 & 0.5 \\
\hline $\mathrm{BM}$ & 34,515 & 200 & 516 & 3.0 & 979.1 & 979.1 & 979.4 & 0.3 \\
\hline $\mathrm{BN}$ & 35,185 & 162 & 408 & 3.8 & 980.2 & 980.2 & 980.3 & 0.1 \\
\hline BO & 35,778 & 73 & 308 & 5.1 & 980.8 & 980.8 & 981.8 & 1.0 \\
\hline BP & 36,015 & 100 & 352 & 4.5 & 981.7 & 981.7 & 982.4 & 0.7 \\
\hline BQ & 36,301 & 125 & 378 & 3.3 & 982.5 & 982.5 & 983.1 & 0.6 \\
\hline BR & 36,935 & 150 & 527 & 2.4 & 983.3 & 983.3 & 983.9 & 0.6 \\
\hline BS & 36,991 & 150 & 629 & 2.0 & 984.1 & 984.1 & 984.9 & 0.8 \\
\hline BT & 37,784 & 125 & 417 & 3.0 & 984.5 & 984.5 & 985.4 & 0.9 \\
\hline $\mathrm{BU}$ & 37,813 & 125 & 549 & 2.3 & 985.4 & 985.4 & 986.3 & 0.9 \\
\hline BV & 38,378 & 100 & 532 & 2.4 & 985.9 & 985.9 & 986.6 & 0.7 \\
\hline BW & 40,484 & 150 & 608 & 2.1 & 986.7 & 986.7 & 987.6 & 0.9 \\
\hline $\mathrm{BX}$ & 40,611 & 150 & 579 & 2.2 & 986.7 & 986.7 & 987.7 & 1.0 \\
\hline BY & 41,373 & 175 & 523 & 2.4 & 987.6 & 987.6 & 988.4 & 0.8 \\
\hline BZ & 41,820 & 125 & 405 & 3.1 & 987.9 & 987.9 & 988.8 & 0.9 \\
\hline CA & 42,001 & 40 & 181 & 7.0 & 988.2 & 988.2 & 989.0 & 0.8 \\
\hline $\mathrm{CB}$ & 42,065 & 44 & 197 & 6.4 & 989.0 & 989.0 & 989.4 & 0.4 \\
\hline $\mathrm{CC}$ & 42,168 & 75 & 380 & 3.3 & 990.0 & 990.0 & 990.1 & 0.1 \\
\hline $\mathrm{CD}$ & 43,167 & 75 & 356 & 3.5 & 990.6 & 990.6 & 991.4 & 0.8 \\
\hline
\end{tabular}

${ }^{1}$ A floodway is defined as the channel of a river or other watercourse and the adjacent land areas that must be reserved to discharge the 1-percent annual exceedance probability flood without increasing the water-surface elevation more than a designated height (Federal Emergency Management Agency, 2020).

${ }^{2}$ Station is the distance in feet upstream from stream mouth.

${ }^{3}$ Backwater elevation from river station 44,302 of the Chippewa Creek model. 
Table 1.4. Floodway data table for the unnamed tributary to Styx River, Wayne County, Ohio.

[\%, percent; AEP, annual exceedance probability; BW, backwater; NAVD 88, North American Vertical Datum of 1988]

\begin{tabular}{|c|c|c|c|c|c|c|c|c|}
\hline \multicolumn{2}{|c|}{ Location } & \multicolumn{3}{|c|}{ Floodway $^{1}$} & \multicolumn{4}{|c|}{$1 \%$ AEP water-surface elevation } \\
\hline $\begin{array}{l}\text { Cross } \\
\text { section } \\
\text { letter }\end{array}$ & $\begin{array}{c}\text { Station }^{2} \\
\text { (feet) }\end{array}$ & $\begin{array}{c}\text { Width, } \\
\text { (feet) }\end{array}$ & $\begin{array}{c}\text { Cross } \\
\text { sectional } \\
\text { area } \\
\text { (square feet) }\end{array}$ & $\begin{array}{l}\text { Mean } \\
\text { velocity } \\
\text { (feet per } \\
\text { second) }\end{array}$ & $\begin{array}{l}\text { Modeled stream with } \\
\text { BW (if any) from } \\
\text { receiving stream } \\
\text { (feet, NAVD 88) }\end{array}$ & $\begin{array}{l}\text { Modeled stream } \\
\text { without BW from } \\
\text { receiving stream } \\
\text { (feet, NAVD 88) }\end{array}$ & $\begin{array}{l}\text { Modeled stream with } \\
\text { floodway without BW } \\
\text { from receiving stream } \\
\text { (feet, NAVD 88) }\end{array}$ & $\begin{array}{c}\text { Increase in water surface } \\
\text { (without BW) } \\
\text { due to } \\
\text { floodway (feet) }\end{array}$ \\
\hline A & 64 & 35 & 222 & 4.2 & ${ }^{3} 956.0$ & 953.7 & 954.5 & 0.8 \\
\hline B & 254 & 40 & 249 & 3.7 & ${ }^{3} 956.0$ & 953.9 & 954.8 & 0.9 \\
\hline $\mathrm{C}$ & 423 & 40 & 186 & 5.0 & ${ }^{3} 956.0$ & 954.3 & 955.1 & 0.8 \\
\hline D & 440 & 40 & 187 & 5.0 & ${ }^{3} 956.0$ & 954.4 & 955.2 & 0.8 \\
\hline E & 642 & 45 & 230 & 4.0 & ${ }^{3} 956.0$ & 955.4 & 955.9 & 0.5 \\
\hline $\mathrm{F}$ & 814 & 40 & 167 & 5.6 & ${ }^{3} 956.0$ & 955.7 & 956.2 & 0.5 \\
\hline G & 842 & 45 & 188 & 5.0 & 956.1 & 956.1 & 956.4 & 0.3 \\
\hline $\mathrm{H}$ & 906 & 50 & 254 & 3.7 & 957.4 & 957.4 & 957.6 & 0.2 \\
\hline I & 1,609 & 35 & 156 & 6.0 & 958.9 & 958.9 & 959.0 & 0.1 \\
\hline $\mathrm{J}$ & 2,007 & 40 & 179 & 5.2 & 960.6 & 960.6 & 960.6 & 0.0 \\
\hline K & 2,212 & 40 & 133 & 7.0 & 961.2 & 961.2 & 961.3 & 0.1 \\
\hline $\mathrm{L}$ & 2,404 & 30 & 128 & 7.3 & 962.6 & 962.6 & 962.6 & 0.0 \\
\hline M & 2,425 & 28 & 138 & 6.7 & 962.9 & 962.9 & 962.9 & 0.0 \\
\hline $\mathrm{N}$ & 2,492 & 28 & 166 & 5.6 & 963.7 & 963.7 & 964.1 & 0.4 \\
\hline $\mathrm{O}$ & 2,546 & 35 & 164 & 5.7 & 964.0 & 964.0 & 964.3 & 0.3 \\
\hline $\mathrm{P}$ & 2,642 & 40 & 178 & 5.2 & 964.2 & 964.2 & 964.6 & 0.4 \\
\hline Q & 2,782 & 45 & 156 & 6.0 & 964.9 & 964.9 & 964.9 & 0.0 \\
\hline $\mathrm{R}$ & 3,134 & 240 & 289 & 3.2 & 965.5 & 965.5 & 966.3 & 0.8 \\
\hline $\mathrm{S}$ & 3,286 & 398 & 436 & 2.2 & 965.8 & 965.8 & 966.7 & 0.9 \\
\hline $\mathrm{T}$ & 3,340 & 335 & 732 & 3.1 & 966.6 & 966.6 & 967.0 & 0.4 \\
\hline $\mathrm{U}$ & 3,577 & 40 & 143 & 3.4 & 967.7 & 967.7 & 967.7 & 0.0 \\
\hline V & 3,774 & 32 & 95 & 5.0 & 967.9 & 967.9 & 968.1 & 0.2 \\
\hline W & 4,078 & 33 & 80 & 6.0 & 969.4 & 969.4 & 969.8 & 0.4 \\
\hline $\mathrm{X}$ & 4,111 & 32 & 90 & 5.3 & 969.9 & 969.9 & 970.2 & 0.3 \\
\hline Y & 4,368 & 30 & 80 & 6.0 & 971.4 & 971.4 & 971.7 & 0.3 \\
\hline Z & 4,766 & 34 & 93 & 5.2 & 974.2 & 974.2 & 974.5 & 0.3 \\
\hline
\end{tabular}


Table 1.4. Floodway data table for the unnamed tributary to Styx River, Wayne County, Ohio.-Continued

[\%, percent; AEP, annual exceedance probability; BW, backwater; NAVD 88, North American Vertical Datum of 1988]

\begin{tabular}{|c|c|c|c|c|c|c|c|c|}
\hline \multicolumn{2}{|c|}{ Location } & \multicolumn{3}{|c|}{ Floodway $^{1}$} & \multicolumn{4}{|c|}{$1 \%$ AEP water-surface elevation } \\
\hline $\begin{array}{l}\text { Cross } \\
\text { section } \\
\text { letter }\end{array}$ & $\begin{array}{c}\text { Station }^{2} \\
\text { (feet) }\end{array}$ & $\begin{array}{c}\text { Width, } \\
\text { (feet) }\end{array}$ & $\begin{array}{c}\text { Cross } \\
\text { sectional } \\
\text { area } \\
\text { (square feet) }\end{array}$ & $\begin{array}{c}\text { Mean } \\
\text { velocity } \\
\text { (feet per } \\
\text { second) }\end{array}$ & $\begin{array}{l}\text { Modeled stream with } \\
\text { BW (if any) from } \\
\text { receiving stream } \\
\text { (feet, NAVD 88) }\end{array}$ & $\begin{array}{l}\text { Modeled stream } \\
\text { without BW from } \\
\text { receiving stream } \\
\text { (feet, NAVD 88) }\end{array}$ & $\begin{array}{l}\text { Modeled stream with } \\
\text { floodway without BW } \\
\text { from receiving stream } \\
\text { (feet, NAVD 88) }\end{array}$ & $\begin{array}{c}\text { Increase in water surface } \\
\text { (without BW) } \\
\text { due to } \\
\text { floodway (feet) }\end{array}$ \\
\hline $\mathrm{AA}$ & 4,966 & 35 & 139 & 6.4 & 976.3 & 976.3 & 976.6 & 0.3 \\
\hline $\mathrm{AB}$ & 5,166 & 37 & 123 & 7.2 & 977.3 & 977.3 & 977.6 & 0.3 \\
\hline $\mathrm{AC}$ & 5,365 & 40 & 137 & 6.5 & 979.0 & 979.0 & 979.1 & 0.1 \\
\hline $\mathrm{AD}$ & 5,567 & 34 & 116 & 7.7 & 980.3 & 980.3 & 980.4 & 0.1 \\
\hline $\mathrm{AE}$ & 5,963 & 35 & 120 & 7.4 & 983.7 & 983.7 & 983.8 & 0.1 \\
\hline $\mathrm{AF}$ & 6,152 & 40 & 164 & 5.4 & 985.0 & 985.0 & 985.2 & 0.2 \\
\hline $\mathrm{AG}$ & 6,416 & 40 & 127 & 7.0 & 985.8 & 985.8 & 986.3 & 0.5 \\
\hline
\end{tabular}

${ }^{1}$ A floodway is defined as the channel of a river or other watercourse and the adjacent land areas that must be reserved to discharge the 1-percent annual exceedance probability flood without increasing the water-surface elevation more than a designated height (Federal Emergency Management Agency, 2020).

${ }^{2}$ Station is the distance in feet upstream from stream mouth.

${ }^{3}$ Backwater elevation from river station 7,295 of the Styx River model. 
Table 1.5. Floodway data table for the unnamed tributary to Styx River overflow, Wayne County, Ohio.

[\%, percent; AEP, annual exceedance probability; BW, backwater; NAVD 88, North American Vertical Datum of 1988]

\begin{tabular}{|c|c|c|c|c|c|c|c|c|}
\hline \multicolumn{2}{|c|}{ Location } & \multicolumn{3}{|c|}{ Floodway ${ }^{1}$} & \multicolumn{4}{|c|}{$1 \%$ AEP water-surface elevation } \\
\hline $\begin{array}{l}\text { Cross } \\
\text { section } \\
\text { letter }\end{array}$ & $\begin{array}{c}\text { Station }^{2} \\
\text { (feet) }\end{array}$ & $\begin{array}{l}\text { Width } \\
\text { (feet) }\end{array}$ & $\begin{array}{c}\text { Cross } \\
\text { sectional } \\
\text { area } \\
\text { (square feet) }\end{array}$ & $\begin{array}{l}\text { Mean } \\
\text { velocity } \\
\text { (feet per } \\
\text { second) }\end{array}$ & $\begin{array}{l}\text { Modeled stream with } \\
\text { BW (if any) from } \\
\text { receiving stream } \\
\text { (feet, NAVD 88) }\end{array}$ & $\begin{array}{l}\text { Modeled stream } \\
\text { without BW from } \\
\text { receiving stream } \\
\text { (feet, NAVD 88) }\end{array}$ & $\begin{array}{l}\text { Modeled stream with } \\
\text { floodway without BW } \\
\text { from receiving stream, } \\
\text { (feet, NAVD 88) }\end{array}$ & $\begin{array}{c}\text { Increase in water surface } \\
\text { (without BW) } \\
\text { due to } \\
\text { floodway (feet) }\end{array}$ \\
\hline A & 374 & 140 & 507 & 0.8 & 967.5 & 967.5 & 967.6 & 0.1 \\
\hline B & 559 & 100 & 335 & 1.2 & 967.5 & 967.5 & 967.6 & 0.1 \\
\hline $\mathrm{C}$ & 846 & 100 & 221 & 1.9 & 967.6 & 967.6 & 967.8 & 0.2 \\
\hline D & 1,121 & 100 & 174 & 2.4 & 967.9 & 967.9 & 968.3 & 0.4 \\
\hline $\mathrm{E}$ & 1,377 & 100 & 138 & 3.0 & 968.9 & 968.9 & 968.9 & 0.0 \\
\hline $\mathrm{F}$ & 1,673 & 100 & 117 & 3.5 & 970.5 & 970.5 & 970.9 & 0.4 \\
\hline G & 1,807 & 100 & 175 & 2.4 & 970.9 & 970.9 & 971.7 & 0.8 \\
\hline $\mathrm{H}$ & 2,154 & 100 & 113 & 3.7 & 972.8 & 972.8 & 973.3 & 0.5 \\
\hline I & 2,392 & 100 & 139 & 3.0 & 975.2 & 975.2 & 975.4 & 0.2 \\
\hline $\mathrm{J}$ & 2,487 & 100 & 199 & 2.1 & 976.0 & 976.0 & 976.3 & 0.3 \\
\hline
\end{tabular}

${ }^{1}$ A floodway is defined as the channel of a river or other watercourse and the adjacent land areas that must be reserved to discharge the 1-percent annual exceedance probability flood without increasing the water-surface elevation more than a designated height (Federal Emergency Management Agency, 2020).

${ }^{2}$ Station is the distance in feet upstream from stream mouth. 
For additional information contact:

Director, Ohio-Kentucky-Indiana Water Science Center U.S. Geological Survey

6460 Busch Blvd., Suite 100

Columbus, $\mathrm{OH} 43229$

614-430-7700

Publishing support provided by the Indianapolis Publishing Service Center 
